\title{
Leptogenesis in the Exceptional Supersymmetric Standard Model: flavour dependent lepton asymmetries
}

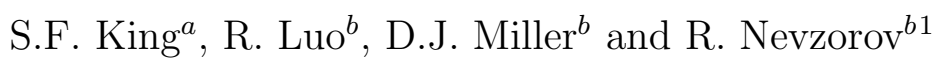 \\ ${ }^{a}$ School of Physics and Astronomy, University of Southampton, \\ Southampton, SO17 1BJ, U.K. \\ ${ }^{b}$ Department of Physics and Astronomy, University of Glasgow, \\ Glasgow G12 8QQ, U.K.
}

\begin{abstract}
We calculate flavour dependent lepton asymmetries within the $E_{6}$ inspired Supersymmetric Standard Model ( $\left.\mathrm{E}_{6} \mathrm{SSM}\right)$, which has an extra $U(1)_{N}$ symmetry. In this model, the right-handed neutrino doesn't participate in gauge interactions, allowing it to be used for both the see-saw mechanism and leptogenesis. Extra Higgs, leptons and leptoquarks predicted by the $\mathrm{E}_{6} \mathrm{SSM}$ contribute to the ordinary lepton $\mathrm{CP}$ asymmetries induced by the decays of the lightest right-handed neutrino (and sneutrino) and give rise to a set of extra decay asymmetries. We find that the CP asymmetries can be relatively large, even when the lightest right-handed neutrino is as light as $10^{6} \mathrm{GeV}$.
\end{abstract}

\footnotetext{
${ }^{1}$ On leave of absence from the Theory Department, ITEP, Moscow, Russia
} 


\section{Introduction}

The baryon asymmetry in the universe $\eta_{B}=\left(n_{B}-n_{\bar{B}}\right) / n_{\gamma}=(6.21 \pm 0.16) \times 10^{-10}$ [1] is one of the motivations to explore physics beyond the elementary particle Standard Model (SM), if the Big Bang nucleosynthesis theory is the right description of the evolution of the universe. Among new physics mechanisms for baryogenesis: are GUT baryogenesis [2], electroweak baryogenesis [3]-[4], baryogenesis via leptogenesis [5]-6], the Affleck-Dine mechanism [7] and so on. Leptogenesis is a particularly interesting mechanism for the generation of baryon asymmetry. It is almost unavoidable in seesaw models [8] because all three Sakharov conditions [9] can be naturally fulfilled in this scenario. In particular, the seesaw mechanism requires that lepton number is violated while complex neutrino Yukawa couplings can provide a source for CP violation. In the simplest realisation of the seesaw mechanism, i.e. in the so-called type I seesaw models, the lepton asymmetry is induced by the heavy right-handed neutrino decays. This lepton asymmetry is partially converted into baryon asymmetry via sphaleron processes [10].

A lot of work has been done in the scenario of the leptogenesis from right-handed neutrino decay. Initially lepton $\mathrm{CP}$ asymmetries which stem from decays of the lightest right-handed neutrino were calculated within the SM [11] and its minimal supersymmetric (SUSY) extension (MSSM) [12] assuming the type I seesaw mechanism of neutrino mass generation. In the early studies of leptogenesis (see for example [13]) flavour effects were ignored. The importance of flavour effects was emphasised in [14], [15]. The process of lepton asymmetry generation in the seesaw models with triplet scalar field and/or triplet fermion field was analysed in [16]-[17]. In the case of Dirac neutrinos leptogenesis was studied in [18].

A potential drawback of supersymmetric thermal leptogenesis is the lower bound on the mass of the lightest right-handed neutrino $M_{1}$. Indeed, it was shown that the appropriate amount of baryon asymmetry in the SM and MSSM can be induced only if $M_{1} \gtrsim 10^{9} \mathrm{GeV}$ [19]. In the framework of supergravity the lower bound on $M_{1}$ leads to the gravitino problem [20] as follows. After inflation the universe thermalizes with a reheat temperature $T_{R}$. If $T_{R}>M_{1}$, right-handed neutrinos are produced by thermal scattering in the reheating epoch. This means that thermal leptogenesis could take place in the MSSM and other SUSY models if $T_{R} \gtrsim 10^{9} \mathrm{GeV}$. At the same time such a high reheating temperature results in an overproduction of gravitinos. Because gravitinos have a long lifetime they may decay during or after Big Bang Nucleosynthesis (BBN) destroying the agreement between the predicted and observed light element abundances. Hence the relic abundance of gravitinos should be constrained from above to preserve the success of BBN. It was argued that the gravitino density becomes low enough when $T_{R} \lesssim 10^{6-7} \mathrm{GeV}$ 
[21]. For such a low reheating temperature and relatively small mass of the lightest righthanded neutrino $M_{1} \sim T e V$ thermal leptogenesis in SUSY models can still be effective if a set of soft supersymmetry breaking terms provides an additional source of both lepton number violation and $\mathrm{CP}$ violation (soft leptogenesis) [22], [17]. TeV scale thermal leptogenesis is also possible if the spectrum of heavy Majorana right-handed neutrinos is quasi-degenerate (so-called resonant leptogenesis [23]) or the particle content of the considered models involves extra particles beyond the SM and/or MSSM [24]. Alternatively, right-handed neutrinos can be produced non-thermally even at very low reheating temperatures, for instance in inflaton decay [25], or in preheating [26]. The gravitino problem can be evaded automatically if the gravitino is the lightest supersymmetric particle in the considered SUSY model [27] or if it is rather heavy so that gravitinos decay before BBN [28].

In this paper, we study the generation of lepton asymmetry in the framework of the Exceptional Supersymmetric Standard Model ( $\mathrm{E}_{6} \mathrm{SSM}$ ) [29]-[30]. This $E_{6}$ inspired SUSY model is based on the low-energy standard model gauge group together with an extra $U(1)_{N}$ gauge symmetry under which right-handed neutrinos have zero charge. In the $\mathrm{E}_{6} \mathrm{SSM}$ the $\mu$ problem is solved in a similar way to the NMSSM, but without the accompanying problems of singlet tadpoles or domain walls. Because right-handed neutrinos do not participate in the gauge interactions in the considered model they may be superheavy, shedding light on the origin of the mass hierarchy in the lepton sector and providing a mechanism for the generation of lepton and baryon asymmetry of the Universe.

Our analysis presented here goes well beyond what has appeared so far in the literature [31]. In this article we calculate lepton CP asymmetries that stem from the decays of the lightest right-handed neutrino taking into account flavour effects. It means that, in contrast with [31, we treat the decay asymmetries associated with different lepton flavours in the final state separately. We also define and compute flavour CP asymmetries originating from the decays of the lightest right-handed neutrino into the exotic leptoquarks (and squarks) that carry lepton and baryon number simultaneouly where our results differ from those in [31]. Finally we perform a comprehensive numerical analysis of the impact of the new particles and interactions appearing in the $\mathrm{E}_{6} \mathrm{SSM}$ on the $\mathrm{CP}$ asymmetries within see-saw models with sequential dominance of right-handed neutrinos [32]-34]. Our results show that it may be possible to achieve successful thermal leptogenesis even with reheat temperature as low as $10^{6} \mathrm{GeV}$.

The paper is organised as follows. In the next section we briefly review the $\mathrm{E}_{6} \mathrm{SSM}$. In section 3 we calculate lepton CP asymmetries within this model. The results of our numerical analysis are discussed in section 4 . Section 5 is reserved for our conclusions and outlook. 


\section{Exceptional SUSY model}

The $\mathrm{E}_{6} \mathrm{SSM}$ is based on the $S U(3)_{C} \times S U(2)_{W} \times U(1)_{Y} \times U(1)_{N}$ gauge group which is a subgroup of $E_{6}$. An additional low energy $U(1)_{N}$, that is not present either in the SM or in the MSSM, is a linear superposition of $U(1)_{\chi}$ and $U(1)_{\psi}$, i.e.

$$
U(1)_{N}=\frac{1}{4} U(1)_{\chi}+\frac{\sqrt{15}}{4} U(1)_{\psi},
$$

where $U(1)_{\psi}$ and $U(1)_{\chi}$ symmetries are defined by:

$$
E_{6} \rightarrow S O(10) \times U(1)_{\psi}, \quad S O(10) \rightarrow S U(5) \times U(1)_{\chi}
$$

To ensure anomaly cancellation the particle content of the $\mathrm{E}_{6} \mathrm{SSM}$ is extended to include three complete fundamental 27 representations of $E_{6}$ at low energies. These multiplets decompose under the $S U(5) \times U(1)_{N}$ subgroup of $E_{6}$ as follows:

$$
\begin{aligned}
27_{i} \rightarrow\left(10, \frac{1}{\sqrt{40}}\right)_{i}+ & \left(5^{*}, \frac{2}{\sqrt{40}}\right)_{i}+\left(5^{*},-\frac{3}{\sqrt{40}}\right)_{i}+\left(5,-\frac{2}{\sqrt{40}}\right)_{i}+ \\
& +\left(1, \frac{5}{\sqrt{40}}\right)_{i}+(1,0)_{i} .
\end{aligned}
$$

The first and second quantities in the brackets are the $S U(5)$ representation and extra $U(1)_{N}$ charge while $i$ is a family index that runs from 1 to 3 . An ordinary SM family which contains the doublets of left-handed quarks $Q_{i}$ and leptons $L_{i}$, right-handed upand down-quarks $\left(u_{i}^{c}\right.$ and $\left.d_{i}^{c}\right)$ as well as right-handed charged leptons, is assigned to $\left(10, \frac{1}{\sqrt{40}}\right)_{i}+\left(5^{*}, \frac{2}{\sqrt{40}}\right)_{i}$. Right-handed neutrinos $N_{i}^{c}$ should be associated with the last term in Eq. (2),$(1,0)_{i}$. The next-to-last term in Eq. (2),$\left(1, \frac{5}{\sqrt{40}}\right)$, represents SM-type singlet fields $S_{i}$ which carry non-zero $U(1)_{N}$ charges and therefore survive down to the EW scale. The pair of $S U(2)_{W}$-doublets $\left(H_{1 i}\right.$ and $\left.H_{2 i}\right)$ that are contained in $\left(5^{*},-\frac{3}{\sqrt{40}}\right)_{i}$ and $\left(5,-\frac{2}{\sqrt{40}}\right)_{i}$ have the quantum numbers of Higgs doublets. So they form either Higgs or inert Higgs $S U(2)_{W}$ multiplets 1 . Other components of these $S U(5)$ multiplets form colour triplets of exotic quarks $\bar{D}_{i}$ and $D_{i}$ with electric charges $-1 / 3$ and $+1 / 3$ respectively. These exotic quark states carry a $B-L$ charge $\left( \pm \frac{2}{3}\right)$ twice larger than that of ordinary ones. Therefore in phenomenologically viable $E_{6}$ inspired models they can be either diquarks or leptoquarks.

In addition to the complete $27_{i}$ multiplets the low energy particle spectrum of the $\mathrm{E}_{6} \mathrm{SSM}$ is supplemented by $S U(2)_{W}$ doublet $H^{\prime}$ and anti-doublet $\bar{H}^{\prime}$ states from extra $27^{\prime}$ and $\overline{27^{\prime}}$ to preserve gauge coupling unification. These components of the $E_{6}$ fundamental

\footnotetext{
${ }^{1}$ We use the terminology "inert Higgs" to denote Higgs like doublets that do not develop vacuum expectation values (VEVs).
} 
representation originate from $\left(5^{*}, \frac{2}{\sqrt{40}}\right)$ of $27^{\prime}$ and $\left(5,-\frac{2}{\sqrt{40}}\right)$ of $\overline{27^{\prime}}$ by construction. The splitting of $27^{\prime}$ and $\overline{27^{\prime}}$ multiplets can be naturally achieved, for example, in the framework of orbifold GUTs [35]. Thus, in addition to a $Z^{\prime}$ corresponding to the $U(1)_{N}$ symmetry, the $\mathrm{E}_{6} \mathrm{SSM}$ involves extra matter beyond the MSSM with the quantum numbers of three $5+5^{*}$ representations of $S U(5)$ plus three $S U(5)$ singlets with $U(1)_{N}$ charges. The presence of a $Z^{\prime}$ boson and exotic quarks predicted by the $\mathrm{E}_{6} \mathrm{SSM}$ provides spectacular new physics signals at the LHC which were discussed in [29]-[30], [36].

As any other supersymmetric model, the $\mathrm{E}_{6} \mathrm{SSM}$ suffers from problems related with rapid proton decay. In other words gauge symmetry does not forbid lepton and baryon number violating operators. Moreover exotic particles in the $E_{6}$ inspired SUSY models give rise to new Yukawa interactions that induce unacceptably large non-diagonal flavour transitions in general. To suppress flavour changing processes in the $\mathrm{E}_{6} \mathrm{SSM}$ an approximate $Z_{2}^{H}$ symmetry is imposed. All superfields except one pair of $H_{1, i}$ and $H_{2, i}$ (say $H_{d} \equiv H_{1,3}$ and $\left.H_{u} \equiv H_{2,3}\right)$ and one SM-type singlet field $\left(S \equiv S_{3}\right)$ are odd under this symmetry. The $Z_{2}^{H}$ symmetry reduces the structure of the Yukawa interactions to:

$$
\begin{gathered}
W_{\mathrm{E}_{6} \mathrm{SSM}} \simeq \lambda S\left(H_{u} H_{d}\right)+\lambda_{\alpha \beta} S\left(H_{1 \alpha} H_{2 \beta}\right)+\kappa_{i j} S\left(D_{i} \bar{D}_{j}\right)+f_{\alpha \beta}\left(H_{d} H_{2 \alpha}\right) S_{\beta} \\
+\tilde{f}_{\alpha \beta}\left(H_{1 \alpha} H_{u}\right) S_{\beta}+h_{i j}^{U}\left(H_{u} Q_{i}\right) u_{j}^{c}+h_{i j}^{D}\left(H_{d} Q_{i}\right) d_{j}^{c}+h_{i j}^{E}\left(H_{d} L_{i}\right) e_{j}^{c}+h_{i j}^{N}\left(H_{u} L_{i}\right) N_{j}^{c} \\
+\frac{1}{2} M_{i j} N_{i}^{c} N_{j}^{c}+\mu^{\prime}\left(L_{4} \bar{L}_{4}\right)+h_{4 j}^{E}\left(H_{d} L_{4}\right) e_{j}^{c}+h_{4 j}^{N}\left(H_{u} L_{4}\right) N_{j}^{c} .
\end{gathered}
$$

where $L_{4} \equiv H^{\prime}, \bar{L}_{4} \equiv \bar{H}^{\prime}, \alpha, \beta=1,2$ and $i, j=1,2,3$. One can notice that the survival components from the $27^{\prime}$ and $\overline{27^{\prime}}$ manifest themselves in the Yukawa interactions (3) as fields with lepton number $L= \pm 1$. Consequently, $L_{4}$ couples to other fields as a fourth family lepton doublet and our notations reflect this. The $S U(2)_{W}$ doublets $H_{u}$ and $H_{d}$, that are even under $Z_{2}^{H}$ symmetry, play the role of Higgs fields generating the masses of quarks and leptons after electroweak symmetry breaking (EWSB). The extra $U(1)_{N}$ gauge symmetry forbids an elementary $\mu$ term in the superpotential of $\mathrm{E}_{6} \mathrm{SSM}$ but allows the interaction of Higgs doublets with the SM-type singlet field $S$. The vacuum expectation value (VEV) of the field $S$ breaks the extra $U(1)_{N}$ symmetry thereby providing an effective $\mu$ term as well as the necessary exotic fermion masses.

The superpotential of the $\mathrm{E}_{6} \mathrm{SSM}$ includes two types of bilinear terms. One of them, $\mu^{\prime} L_{4} \bar{L}_{4}$, is solely responsible for the masses of the charged and neutral components of $L_{4}$ and $\bar{L}_{4}$. The corresponding mass term is not suppressed by $E_{6}$ and is not involved in the process of EWSB. Therefore the parameter $\mu^{\prime}$ remains arbitrary. Recent analysis revealed that gauge coupling unification in the $\mathrm{E}_{6} \mathrm{SSM}$ is consistent with $\mu^{\prime}$ around $100 \mathrm{TeV}$ 37. Another type of bilinear terms $\frac{1}{2} M_{i j} N_{i}^{c} N_{j}^{c}$, determines the spectrum of the right-handed neutrinos. These mass terms are forbidden by $E_{6}$ and can be generated only after its 
breakdown [38]. Suppose $N_{H}^{c}$ and $\bar{N}_{H}^{c}$ are components of some extra $27_{H}$ and $\overline{27}_{H}$ representations which develop VEVs along the $D$-flat direction $\left\langle N_{H}^{c}\right\rangle=\left\langle\bar{N}_{H}^{c}\right\rangle \simeq \Lambda$. Then the right-handed neutrino mass terms can be induced through the non-renormalisable interactions of $27_{i}$ and $\overline{27}_{H}$ of the form $\frac{\eta_{i j}}{M_{P l}}\left(\overline{27}_{H} 27_{i}\right)\left(\overline{27}_{H} 27_{j}\right)$. As a result right-handed neutrinos gain masses $M_{i}$ of the order of $\frac{\Lambda^{2}}{M_{P l}}<<M_{X}$. At the same time we assume that $M_{i}$ are much larger than $\mu^{\prime}$ so that the right-handed neutrinos can decay either to a Higgs particle and a fermion component of $L_{4}$ or to a higgsino and a scalar component of $L_{4}$.

Although $Z_{2}^{H}$ eliminates any problem related with non-diagonal flavour transitions it also forbids all Yukawa interactions that would allow the exotic quarks to decay. Since models with stable charged exotic particles are ruled out by various experiments [39] the $Z_{2}^{H}$ symmetry can only be an approximate one. But the breakdown of $Z_{2}^{H}$ should not give rise to operators leading to rapid proton decay. There are two ways to overcome this problem. The resulting Lagrangian has to be invariant either with respect to an exact $Z_{2}^{L}$ symmetry, under which all superfields except lepton ones are even (Model I), or with respect to an exact $Z_{2}^{B}$ discrete symmetry, under which exotic quark and lepton superfields are odd whereas the others remain even (Model II). If the Lagrangian is invariant under the $Z_{2}^{B}$ symmetry transformations then exotic quarks are leptoquarks. If $Z_{2}^{L}$ is imposed then the baryon number conservation requires the exotic quarks to be diquarks. The breakdown of $Z_{2}^{H}$ symmetry also leads to the new interactions of the right-handed neutrinos with exotic particles. The corresponding terms in the superpotential of the $\mathrm{E}_{6} \mathrm{SSM}$ are given by

$$
\Delta W=\xi_{\alpha i j}\left(H_{2 \alpha} L_{i}\right) N_{j}^{c}+\xi_{\alpha 4 j}\left(H_{2 \alpha} L_{4}\right) N_{j}^{c}+g_{k i j}^{N} D_{k} d_{i}^{c} N_{j}^{c}
$$

The Yukawa couplings $g_{i j k}^{N}$ vanish if exotic quarks are diquarks and may have non-zero values if exotic quarks are leptoquarks. Because $Z_{2}^{H}$ symmetry violating interactions may give an appreciable contribution to the amplitude of $K^{0}-\bar{K}^{0}$ oscillations and give rise to new muon decay channels like $\mu \rightarrow e^{-} e^{+} e^{-}$the Yukawa couplings of the related terms are required to be small $\left(\lesssim 10^{-3}-10^{-4}\right)$. This suggests that $\xi_{\alpha i j}$ should also be similarly small, but does not provide any constraint on the couplings $\xi_{\alpha 4 j}$.

Combining the appropriate terms in Eqs. (3) and (4) one can write the part of the superpotential describing the interactions of the right-handed neutrinos with other bosons and fermions in the following compact form:

$$
W_{N}=h_{k x j}^{N}\left(H_{k}^{u} L_{x}\right) N_{j}^{c}+g_{k i j}^{N} D_{k} d_{i}^{c} N_{j}^{c}
$$

where $H_{3}^{u} \equiv H_{u}$ is the usual Higgs doublet, $H_{\alpha}^{u} \equiv H_{2 \alpha}$ are the two extra inert Higgs doublets, $L_{4}$ is the extra lepton doublet, while $D_{k}$ are the exotic quarks. Note that the 
indices run over the following ranges $x=1,2,3,4$ while $k, i, j=1,2,3$. In the Model I $g_{k i j}^{N}=0$ so the only extra particles present are inert Higgs and the fourth lepton doublet, whereas in the Model II all terms which appear on the right-hand side of Eq. (55) including extra leptoquarks can be present.

\section{Decay asymmetries in the $\mathrm{E}_{6} \mathrm{SSM}$}

\subsection{CP asymmetries for the Model I}

In this subsection we discuss Model I corresponding to the case of additional inert Higgs and a fourth lepton family only. The case of additional leptoquarks is considered in the next subsection.

In models with heavy right-handed neutrinos lepton asymmetry can be dynamically generated and then get converted into a baryon asymmetry due to $(B+L)$-violating sphaleron interactions. The generation of lepton asymmetry occurs via the out-of equilibrium decay of the lightest right-handed neutrino $N_{1}$. The process of the lepton asymmetry generation is controlled by the flavour CP (decay) asymmetries $\varepsilon_{1, \ell_{k}}$ that appear on the right-hand side of Boltzmann equations. In the SM there are three decay asymmetries associated with three lepton flavours $e, \mu$ and $\tau$. They are given by

$$
\varepsilon_{1, \ell_{k}}=\frac{\Gamma_{N_{1} \ell_{k}}-\Gamma_{N_{1} \bar{\ell}_{k}}}{\sum_{m}\left(\Gamma_{N_{1} \ell_{m}}+\Gamma_{N_{1} \bar{\ell}_{m}}\right)} .
$$

where $\Gamma_{N_{1} \ell_{k}}$ and $\Gamma_{N_{1} \bar{\ell}_{k}}$ are partial decay widths of $N_{1} \rightarrow L_{k}+H_{u}$ and $N_{1} \rightarrow \bar{L}_{k}+H_{u}^{*}$ with $k, m=1,2,3$. At the tree level CP asymmetries (6) vanish because $\Gamma_{N_{1} \ell_{k}}=\Gamma_{N_{1} \bar{\ell}_{k}}$. If $\mathrm{CP}$ invariance is broken in the lepton sector the non-zero contributions to the $\mathrm{CP}$ asymmetries arise from the interference between the tree-level amplitudes of the lightest right-handed neutrino decays and one-loop corrections to them.

Supersymmetry gives rise to new channels of right-handed neutrino decay into sleptons $\widetilde{L}_{k}$ and Higgsino $\widetilde{H}_{u}$ that also contribute to the generation of total lepton asymmetry. The corresponding flavour CP asymmetries are defined as

$$
\varepsilon_{1, \widetilde{\ell}_{k}}=\frac{\Gamma_{N_{1} \widetilde{\ell}_{k}}-\Gamma_{N_{1} \widetilde{\ell}_{k}^{*}}}{\sum_{m}\left(\Gamma_{N_{1} \widetilde{\ell}_{m}}+\Gamma_{N_{1} \widetilde{\ell}_{m}^{*}}\right)} .
$$

In addition supersymmetry predicts the existence of a scalar partner of the right-handed neutrino $\widetilde{N}_{1}$ (right-handed sneutrino). The decays of the right-handed sneutrino into lepton and Higgsino and into slepton and Higgs provide another important origin of lepton asymmetry. The right-handed sneutrino $\mathrm{CP}$ asymmetries can be determined similarly to 
the neutrino ones

$$
\varepsilon_{\widetilde{1}, \ell_{k}}=\frac{\Gamma_{\widetilde{N}_{1}^{*} \ell_{k}}-\Gamma_{\widetilde{N}_{1} \bar{\ell}_{k}}}{\sum_{m}\left(\Gamma_{\widetilde{N}_{1}^{*} \ell_{m}}+\Gamma_{\widetilde{N}_{1} \bar{\ell}_{m}}\right)}, \quad \varepsilon_{\widetilde{1}, \widetilde{\ell}_{k}}=\frac{\Gamma_{\widetilde{N}_{1} \widetilde{\ell}_{k}}-\Gamma_{\widetilde{N}_{1}^{*} \widetilde{\ell}_{k}^{*}}}{\sum_{m}\left(\Gamma_{\widetilde{N}_{1} \widetilde{\ell}_{m}}+\Gamma_{\widetilde{N}_{1}^{*} \widetilde{\ell}_{m}^{*}}\right)} .
$$

The direct computation of decay asymmetries in SUSY models reveals that

$$
\varepsilon_{1, \ell_{k}}=\varepsilon_{1, \widetilde{\ell}_{k}}=\varepsilon_{\widetilde{1}, \ell_{k}}=\varepsilon_{\widetilde{1}, \widetilde{\ell}_{k}} .
$$

In the Exceptional SUSY model the relation between different types of decay asymmetries (91) remains intact. But extra particles predicted by the $\mathrm{E}_{6} \mathrm{SSM}$ result in the new channels of the decays of right-handed neutrino and its superpartner. In the $\mathrm{E}_{6} \mathrm{SSM}$ Model I only inert Higgs superfields and $L_{4}$ are allowed to have non-zero Yukawa couplings to the right-handed neutrino superfields (see Eq. (5)). Since the extra inert Higgs and the fourth family of (vector-like) leptons are expected to be significantly lighter than $N_{1}$ these Yukawa interactions induce new decay modes of the lightest right-handed neutrino and sneutrino. A complete set of possible decay channels of the lightest right-handed neutrino and sneutrino includes

$$
N_{1} \rightarrow L_{x}+H_{k}^{u}, \quad N_{1} \rightarrow \widetilde{L}_{x}+\widetilde{H}_{k}^{u}, \quad \widetilde{N}_{1} \rightarrow \bar{L}_{x}+\overline{\widetilde{H}}_{k}^{u}, \quad \widetilde{N}_{1} \rightarrow \widetilde{L}_{x}+H_{k}^{u},
$$

where index $x$ changes from 1 to 4 . At the tree level the rates of these decay modes of $N_{1}$ and $\widetilde{N}_{1}$ are set by the Yukawa couplings $h_{k x 1}^{N}$. Supersymmetry implies that

$$
\Gamma_{N_{1} \ell_{x}}^{k}+\Gamma_{N_{1} \bar{\ell}_{x}}^{k}=\Gamma_{N_{1} \widetilde{\ell}_{x}}^{k}+\Gamma_{N_{1} \widetilde{\ell}_{x}^{*}}^{k}=\Gamma_{\widetilde{N}_{1}^{*} \ell_{x}}^{k}=\Gamma_{\widetilde{N}_{1} \bar{\ell}_{x}}^{k}=\Gamma_{\widetilde{N}_{1} \widetilde{\ell}_{x}}^{k}=\Gamma_{\widetilde{N}_{1}^{*} \widetilde{\ell}_{x}^{*}}^{k}=\frac{\left|h_{k x 1}^{N}\right|^{2}}{8 \pi} M_{1},
$$

where superscript $k$ represents either Higgs (Higgsino) if $k=3$ or inert Higgs (inert Higgsino) field if $k=1,2$ in the final state. Here and further we work in a field basis where the charged lepton Yukawa matrix and mass matrix of the right-handed neutrinos are diagonal. We also assume that supersymmetry breaking scale is negligibly small as compared with $M_{1}$. As a consequence all soft SUSY breaking terms can be safely ignored in our calculations of decay asymmetries and rates.

Each decay channel (10) gives rise to the CP asymmetry that contributes to the generation of total lepton asymmetry. In the considered case the definition of the CP asymmetries (6) coming from the decays of the lightest right-handed neutrino can be generalised in the following way

$$
\varepsilon_{1, f}^{k}=\frac{\Gamma_{N_{1} f}^{k}-\Gamma_{N_{1} \bar{f}}^{k}}{\sum_{m, f^{\prime}}\left(\Gamma_{N_{1} f^{\prime}}^{m}+\Gamma_{N_{1} \bar{f}^{\prime}}^{m}\right)}
$$

where $f$ and $f^{\prime}$ may be either $\ell_{x}$ or $\tilde{\ell}_{x}$ while $\bar{f}$ and $\bar{f}^{\prime}$ should be associated with either $\bar{\ell}_{x}$ or $\tilde{\ell}_{x}^{*}$. Here $\varepsilon_{1, \ell_{n}}^{3}$ and $\varepsilon_{1, \widetilde{\ell}_{n}}^{3}(\mathrm{n}=1,2,3)$ are flavour CP asymmetries that stem from the decays of 


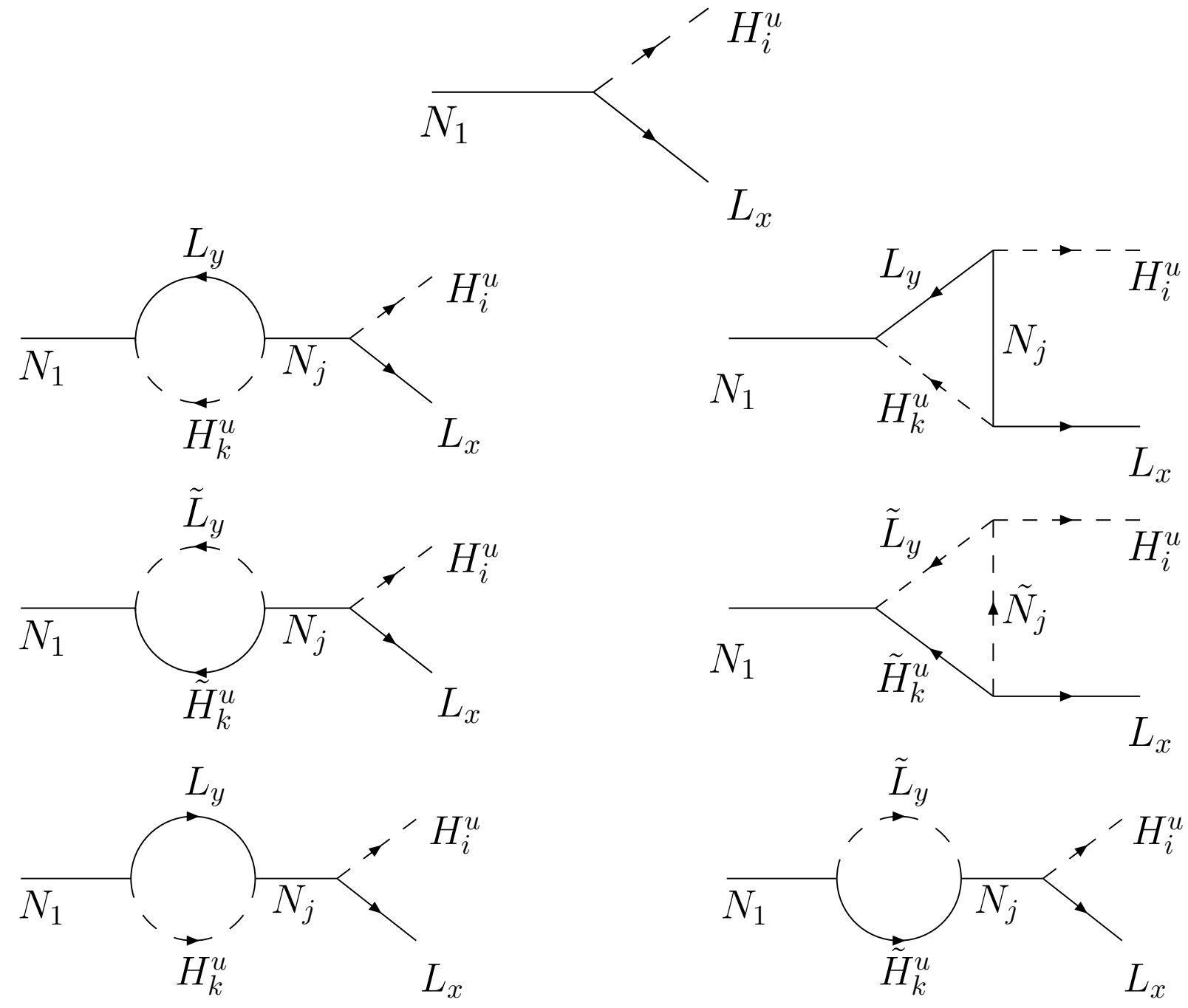

Figure 1: Diagrams that give contribution to the CP asymmetries in the $\mathrm{E}_{6} \mathrm{SSM}$ Model I, including the presence of two extra inert Higgs doublets, and the fourth family lepton doublet.

the lightest right-handed neutrino into leptons (sleptons) and Higgs doublet $H_{u}$ (Higgsino $\left.\widetilde{H}_{u}\right)$ while $\varepsilon_{1, \ell_{4}}^{3}, \varepsilon_{1, \widetilde{\ell}_{4}}^{3}, \varepsilon_{1, f}^{1}$ and $\varepsilon_{1, f}^{2}$ are extra CP asymmetries caused by the new decay channels of $N_{1}$. The denominators of Eqs. (12) contain a sum of partial decay widths of the lightest right-handed neutrino. For $\varepsilon_{1, \ell_{x}}^{k}$ this sum includes all possible partial widths of the decays of $N_{1}$ whose final state involves leptons and fermion components of $L_{4}$. The expressions for $\varepsilon_{1, \widetilde{\ell}_{x}}^{k}$ contain in the denominator a sum of partial decay widths of $N_{1}$ over all possible decay modes that have either slepton or scalar components of $L_{4}$ in the final state. The CP asymmetries caused by the decays of the lightest right-handed sneutrino $\varepsilon_{\widetilde{1}, f}^{k}$ can be defined similarly to the neutrino ones. In this case the right-handed neutrino field in Eqs. (12) ought to be replaced by either $\widetilde{N}_{1}$ or $\widetilde{N}_{1}^{*}$.

As in the SM and MSSM the CP asymmetries within the $\mathrm{E}_{6} \mathrm{SSM}$ Model I arise due to the interference between the tree-level amplitudes of the lightest right-handed neutrino 
decays and the vertex and self-energy corrections to them. The corresponding tree-level and one-loop diagrams are shown in Fig. 1. After the calculation of one-loop diagrams we get

$$
\begin{aligned}
\varepsilon_{1, \ell_{x}}^{k} & =\varepsilon_{1, \tilde{\ell}_{x}}^{k}=\varepsilon_{\widetilde{1}, \ell_{x}}^{k}=\varepsilon_{\widetilde{1}, \tilde{\ell}_{x}}^{k}=\frac{1}{4 \pi A_{1}} \sum_{j=2,3} \operatorname{Im}\left\{A_{j} h_{k x 1}^{N *} h_{k x j}^{N} f^{S}\left(\frac{M_{j}^{2}}{M_{1}^{2}}\right)\right. \\
& \left.+\sum_{m, y} h_{m y 1}^{N *} h_{m x j}^{N} h_{k y j}^{N} h_{k x 1}^{N *} f^{V}\left(\frac{M_{j}^{2}}{M_{1}^{2}}\right)\right\},
\end{aligned}
$$

where

$$
\begin{gathered}
A_{j}=\sum_{m, y}\left(h_{m y 1}^{N *} h_{m y j}^{N}+\frac{M_{1}}{M_{j}} h_{m y 1}^{N} h_{m y j}^{N *}\right), \\
f^{S}(z)=\frac{2 \sqrt{z}}{1-z}, \quad f^{V}(z)=-\sqrt{z} \ln \left(\frac{1+z}{z}\right),
\end{gathered}
$$

with $k, m=1,2,3$ and $x, y=1,2,3,4$. The terms in the right-hand side of Eq. (13) which are proportional to $A_{j}$ are induced by the self-energy diagrams while all other terms come from vertex corrections. It is worth to notice here that the coefficients in front of $f^{S}(x)$ and $f^{V}(x)$ are not the same, in contrast to the simplest realisations of Fukugita-Yanagida mechanism in the SM and MSSM. It means that in general vertex and self-energy contributions to $\varepsilon_{1, f}$ and $\varepsilon_{\widetilde{1}, f}$ are not related to each other in the considered model. This is a common feature of the models in which right-handed Majorana neutrinos interact with a few lepton doublets and with a few doublets that have quantum numbers of Higgs fields.

Because inert Higgs and inert Higgsino fields do not carry any lepton number it is convenient to define the overall $\mathrm{CP}$ asymmetries which are associated with each flavour, i.e.

$$
\varepsilon_{1, f}^{t o t}=\sum_{k} \varepsilon_{1, f}^{k}, \quad \varepsilon_{\widetilde{1}, f}^{t o t}=\sum_{k} \varepsilon_{\widetilde{1}, f}^{k}
$$

These overall decay asymmetries enter in the right-hand side of Boltzmann equations that describe the evolution of lepton number densities. The CP asymmetries (14) can be written in a compact form

$$
\varepsilon_{1, f}^{t o t}=\varepsilon_{\widetilde{1}, f}^{t o t}=\frac{1}{8 \pi\left(\operatorname{Tr} \Pi^{1}\right)} \sum_{j=2,3} \operatorname{Im}\left\{A_{j} \Pi_{f f}^{j} f^{S}\left(\frac{M_{j}^{2}}{M_{1}^{2}}\right)+\left(\Pi^{j}\right)_{f f}^{2} f^{V}\left(\frac{M_{j}^{2}}{M_{1}^{2}}\right)\right\},
$$

where

$$
\Pi_{\ell_{y} \ell_{x}}^{j}=\Pi_{\tilde{\ell}_{y} \tilde{\ell}_{x}}^{j}=\sum_{m} h_{m y 1}^{N *} h_{m x j}^{N}
$$

are three $4 \times 4$ matrices and $A_{j}=\operatorname{Tr} \Pi^{j}+\frac{M_{1}}{M_{j}} \operatorname{Tr} \Pi^{j *}$. Eqs. (15)-(16) indicate that despite a large number of new couplings appeared due to the breakdown of $Z_{H}^{2}$ symmetry only 
some combinations of them contribute to the generation of lepton asymmetries. The parametrisation of the overall flavour $\mathrm{CP}$ asymmetries presented above can be used in any model in which lightest right-handed neutrino can decay into a few lepton multiplets and a few $S U(2)_{W}$ doublets that have quantum numbers of Higgs fields.

In the case of unbroken $Z_{2}^{H}$ symmetry the analytical expressions for the decay asymmetries (13) and (15) are simplified dramatically. In particular, CP asymmetries $\varepsilon_{1, f}^{1}$ and $\varepsilon_{1, f}^{2}$ which are associated with the decays of $N_{1}$ into either scalar or fermion component of inert Higgs superfields $H_{2 \alpha}$ vanish when $Z_{2}^{H}$ symmetry violating Yukawa couplings tend to zero. The analytical expressions for other decay asymmetries reduce to

$$
\begin{gathered}
\varepsilon_{1, \ell_{x}}^{3}=\varepsilon_{1, \widetilde{\ell}_{x}}^{3}=\varepsilon_{\widetilde{1}, \ell_{x}}^{3}=\varepsilon_{\widetilde{1}, \widetilde{\ell}_{x}}^{3}=\frac{1}{8 \pi} \frac{\sum_{j=2,3} \operatorname{Im}\left[h_{3 x 1}^{N *} B_{1 j} h_{3 x j}^{N}\right]}{\sum_{y}\left|h_{3 y 1}^{N}\right|^{2}}, \\
B_{1 j}=\sum_{y}\left\{h_{3 y 1}^{N *} h_{3 y j}^{N} g\left(\frac{M_{j}^{2}}{M_{1}^{2}}\right)+\frac{M_{1}}{M_{j}} h_{3 y 1}^{N} h_{3 y j}^{N *} f^{S}\left(\frac{M_{j}^{2}}{M_{1}^{2}}\right)\right\}, \\
g(z)=f^{V}(z)+f^{S}(z)=\sqrt{z}\left[\frac{2}{1-z}-\ln \left(\frac{1+z}{z}\right)\right],
\end{gathered}
$$

where $x$ and $y$ vary from 1 to 4 . If the second lightest and heaviest right-handed neutrinos are significantly heavier than the lightest one, i.e. $M_{2}, M_{3} \gg M_{1}$, the formulae for the CP asymmetries (17) are simplified even further

$$
\varepsilon_{1, \ell_{x}}^{3} \simeq-\frac{3}{8 \pi} \sum_{j=2,3} \frac{\operatorname{Im}\left[\left(h^{N \dagger} h^{N}\right)_{1 j} h_{3 x 1}^{N *} h_{3 x j}^{N}\right]}{\left(h^{N \dagger} h^{N}\right)_{11}} \frac{M_{1}}{M_{j}},
$$

where $\left(h^{N \dagger} h^{N}\right)_{1 j}=\sum_{y} h_{3 y 1}^{N *} h_{3 y j}^{N}$. From Eq. (17) one can see that the self-energy contribution to the flavour CP asymmetries is twice larger than the vertex one in the considered case.

The derived analytical expressions for the CP asymmetries (17)-(18) are very similar to the MSSM ones. Moreover when Yukawa couplings $h_{34 j}^{N} \rightarrow 0$ extra CP asymmetries induced by the decays

$$
N_{1} \rightarrow L_{4}+H_{u}, \quad N_{1} \rightarrow \widetilde{L}_{4}+\widetilde{H}_{u}, \quad \widetilde{N}_{1} \rightarrow \bar{L}_{4}+\widetilde{H}_{u}, \quad \widetilde{N}_{1} \rightarrow \widetilde{L}_{4}+H_{u},
$$

go to zero and the results for the flavour lepton decay asymmetries obtained within the MSSM are reproduced. However if Yukawa couplings $h_{34 j}^{N}$ have non-zero values the process of generation of lepton asymmetry in the MSSM and $\mathrm{E}_{6} \mathrm{SSM}$ with unbroken $Z_{2}^{H}$ can be entirely different because of the presence of superfields $L_{4}$ in the particle spectrum of the $\mathrm{E}_{6} \mathrm{SSM}$. Indeed, since $h_{34 j}^{N}$ can be either of the order of or even larger than the Yukawa couplings of the ordinary lepton superfields to the Higgs doublet $H_{u}$ the decay rates and $\mathrm{CP}$ asymmetries associated with the decays (19) can be substantially bigger than other 

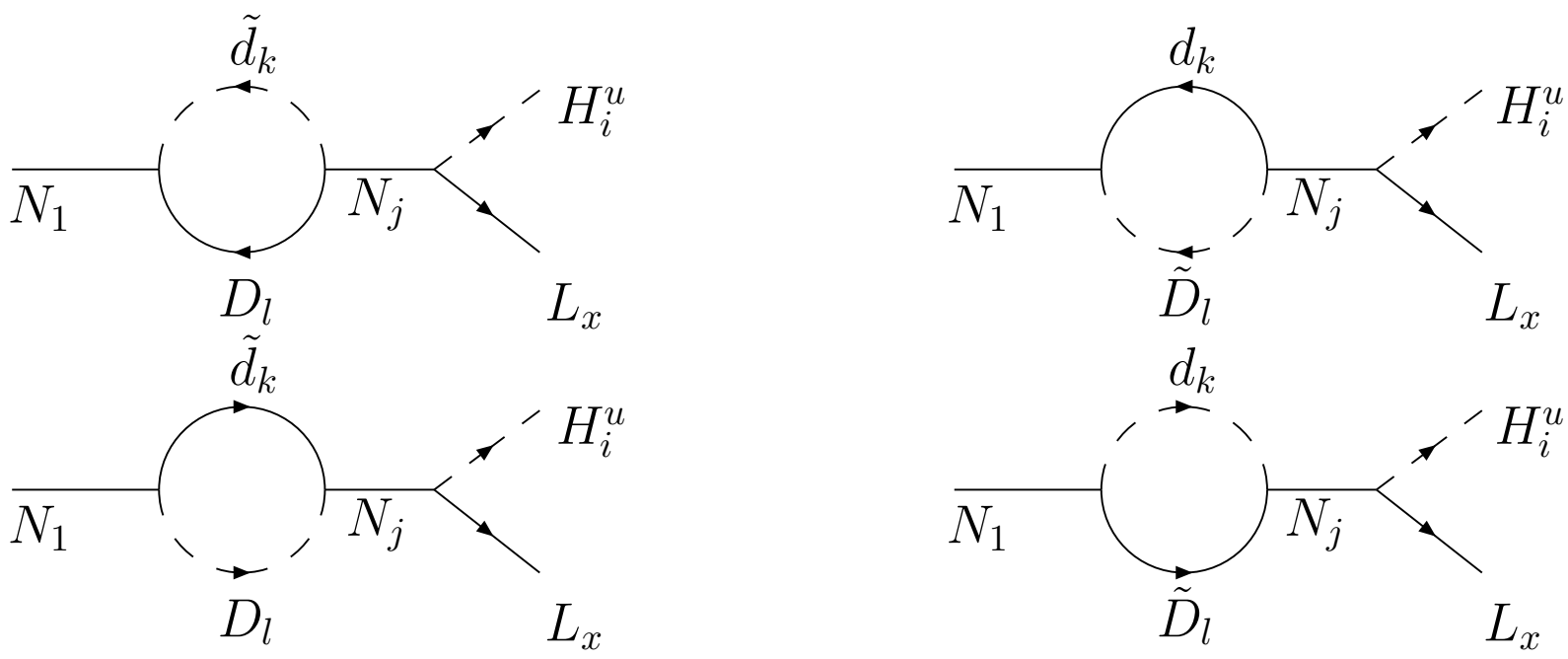

Figure 2: Extra one-loop diagrams involving internal leptoquarks $D$ that contribute to the CP asymmetries associated with the decays $N_{1} \rightarrow L_{x}+H_{k}^{u}$ in the $\mathrm{E}_{6} \mathrm{SSM}$ Model II

decay rates and asymmetries. The fermion and scalar components of the supermultiplet $L_{4}$ being produced in the decays of the lightest right-handed neutrino and sneutrino sequentially decay either to the leptons or to the sleptons changing the induced lepton number asymmetries.

\subsection{CP asymmetries for the Model II}

In the $\mathrm{E}_{6} \mathrm{SSM}$ Model II there are, in addition to the states in Model I, exotic leptoquarks which carry baryon and lepton numbers simultaneously. In this case quark-lepton couplings of $\bar{D}_{i}$ and $D_{i}$ in the superpotential do not violate either baryon or lepton $U(1)$ global symmetries so that these interactions are allowed from the phenomenological point of view. On the other hand these couplings violate $Z_{2}^{H}$ symmetry and therefore the corresponding interactions should be rather weak.

The non-zero complex Yukawa couplings of the leptoquarks to the right-handed Majorana neutrinos (see Eq.(4)) give rise to extra contributions to the CP asymmetries which correspond to different lepton flavours. These contributions come from the one-loop selfenergy diagrams shown in Fig. 2 that contain virtual (possibly exotic) quarks and squarks. Because Yukawa couplings of the leptoquarks do not induce any one-loop vertex corrections to the amplitude of the decay of the lightest right-handed neutrino, lepton decay asymmetries can be described by Eqs. (13) in which $A_{2}$ and $A_{3}$ should be replaced by $\widetilde{A}_{2}$ and $\widetilde{A}_{3}$ where

$$
\widetilde{A}_{j}=A_{j}+\frac{3}{2} \sum_{m, n}\left(g_{m n 1}^{N *} g_{m n j}^{N}+\frac{M_{1}}{M_{j}} g_{m n 1}^{N} g_{m n j}^{N *}\right) .
$$


At the same time the interactions of $D_{i}$ and $\bar{D}_{i}$ with $N_{1}$ and quark superfields give rise to the new channels of the lightest right-handed neutrino and sneutrino decays

$$
N_{1} \rightarrow D_{k}+\widetilde{d}_{i}^{c}, \quad N_{1} \rightarrow \widetilde{D}_{k}+d_{i}^{c}, \quad \widetilde{N}_{1} \rightarrow \bar{D}_{k}+d_{i}, \quad \widetilde{N}_{1} \rightarrow \widetilde{D}_{k}+\widetilde{d}^{c}{ }_{i}
$$

where $D_{k}$ and $\widetilde{D}_{k}$ are fermion and scalar components of leptoquark superfields while $d_{i}$ and $\widetilde{d}_{i}$ are right-handed down type quarks and their superpartners. When the supersymmetry breaking scale lies considerably lower than the lightest right-handed neutrino mass $M_{1}$, the corresponding partial decay widths are determined by the $Z_{2}^{H}$ symmetry violating Yukawa couplings $g_{k i 1}^{N}$ only, i.e.

$$
\begin{gathered}
\Gamma_{N_{1} D_{k}}^{i}+\Gamma_{N_{1} \bar{D}_{k}}^{i}=\Gamma_{N_{1} \widetilde{D}_{k}}^{i}+\Gamma_{N_{1} \widetilde{D}_{k}^{*}}^{i}=\Gamma_{\widetilde{N}_{1}^{*} D_{k}}^{i}=\Gamma_{\widetilde{N}_{1} \bar{D}_{k}}^{i}= \\
=\Gamma_{\widetilde{N}_{1} \widetilde{D}_{k}}^{i}=\Gamma_{\widetilde{N}_{1}^{*} \widetilde{D}_{k}^{*}}^{i}=\frac{3\left|g_{k i 1}^{N}\right|^{2}}{16 \pi} M_{1} .
\end{gathered}
$$

New channels of the decays of the lightest right-handed neutrino (or sneutrino) contribute to the generation of lepton asymmetry via the sequential decay of leptoquarks and their superpartners at low energies. Due to the lepton number conservation, each $D_{k}$ and $\widetilde{D}_{k}$ produce a lepton in the final state whereas the decay of their antiparticles leads to the appearance of an antilepton. As a consequence one can calculate lepton CP asymmetries associated with each additional channel of the lightest right-handed neutrino (or sneutrino) decay (21). We define the CP asymmetries caused by the decays of $N_{1}$ into the exotic quarks (squarks) as follows

$$
\varepsilon_{1, q_{k}}^{i}=\frac{\Gamma_{N_{1} q_{k}}^{i}-\Gamma_{N_{1} \bar{q}_{k}}^{i}}{\sum_{j, m}\left(\Gamma_{N_{1} q_{m}}^{j}+\Gamma_{N_{1} \bar{q}_{m}}^{j}\right)} .
$$

In Eq. (23) $q_{k}$ can be either leptoquark fermion fields $D_{k}$ or their scalar superpartners $\widetilde{D}_{k}$ whereas $\bar{q}_{k}$ represents charge conjugate states $\bar{D}_{k}$ or $\widetilde{D}_{k}^{*}$. The superscripts $i$ and $j$ indicate the generation number of the down type quark or its superpartner in the final state. In the denominator of Eq. (23) we sum over possible partial widths of the decays of $N_{1}$ either into exotic quark and right-handed down type squark if $\varepsilon_{1, q_{k}}^{i}=\varepsilon_{1, D_{k}}^{i}$ or into exotic squark and ordinary $d$-quark if $\varepsilon_{1, q_{k}}^{i}=\varepsilon_{1, \widetilde{D}_{k}}^{i}$. The CP asymmetries $\varepsilon_{\widetilde{1}, q_{k}}^{i}$ which originate from the decays of the lightest right-handed sneutrino into the exotic quark (squark) can be defined in a similar way replacing $N_{1}$ in Eq. (23) by either $\tilde{N}_{1}$ or $\widetilde{N}_{1}^{*}$. It is worth noticing that here we treat the CP asymmetries for the right-handed neutrino (sneutrino) decays to leptons and leptoquarks separately. In other words we do not combine together all possible partial widths of the decays of $N_{1}$ into exotic quarks (squark) and leptons (sleptons) in the denominator of Eq. (23) because leptoquarks and lepton fields carry different quantum numbers.

In the tree level approximation, the CP asymmetries which are associated with the new decay modes of $N_{1}$ and $\widetilde{N}_{1}$ (21) vanish. The non-zero values of $\varepsilon_{1, q_{k}}^{i}$ are induced 

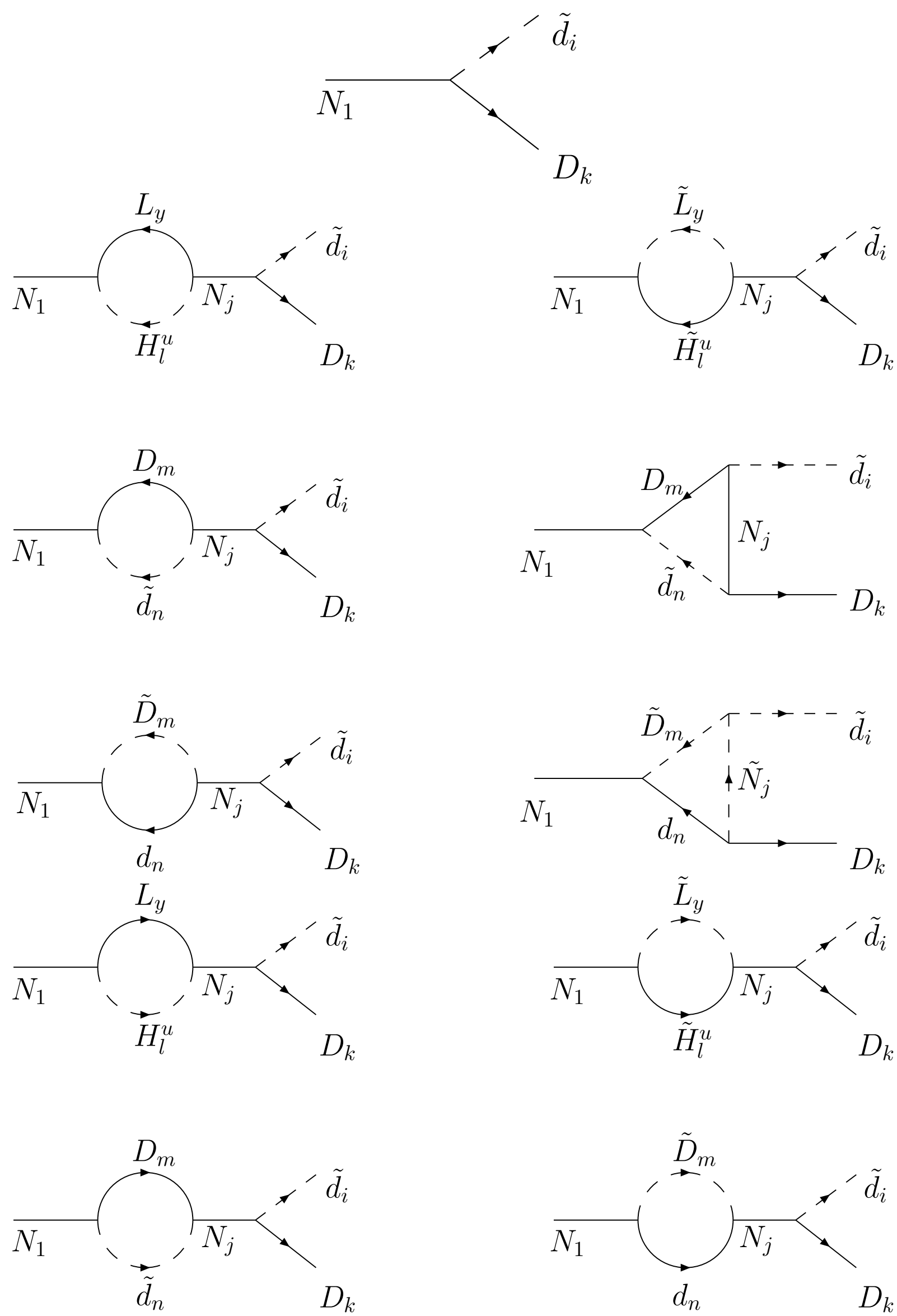

Figure 3: Tree-level and one-loop diagrams that give contribution to the $\mathrm{CP}$ asymmetries associated with the decays $N_{1} \rightarrow D_{k}+d_{i}$ involving final state leptoquarks $D$ in the $\mathrm{E}_{6} \mathrm{SSM}$ Model II. 
after the inclusion of one-loop vertex and self-energy corrections to the decay amplitudes of $N_{1}$ and $\widetilde{N}_{1}$ if some of the Yukawa couplings of the right-handed Majorana neutrinos to leptons and quarks are complex. The tree-level and one-loop diagrams that contribute to the decay asymmetries (23) are presented in Fig. 3. The interference of the corresponding tree-level decay amplitude with the one-loop corrections yields

$$
\begin{aligned}
\varepsilon_{1, D_{k}}^{i} & =\varepsilon_{1, \widetilde{D}_{k}}^{i}=\varepsilon_{\widetilde{1}_{,} D_{k}}^{i}=\varepsilon_{\widetilde{1}, \widetilde{D}_{k}}^{i}=\frac{1}{8 \pi A_{0}} \sum_{j=2,3} \operatorname{Im}\left\{\widetilde{A}_{j} g_{k i j}^{N} g_{k i 1}^{N *} f^{S}\left(\frac{M_{j}^{2}}{M_{1}^{2}}\right)\right. \\
& \left.+\sum_{m, n} g_{m n 1}^{N *} g_{m i j}^{N} g_{k n j}^{N} g_{k i 1}^{N *} f^{V}\left(\frac{M_{j}^{2}}{M_{1}^{2}}\right)\right\},
\end{aligned}
$$

where $A_{0}=\sum_{k, i} g_{k i 1}^{N} g_{k i 1}^{N *}$. As before, supersymmetry ensures that the CP asymmetries originating from the decays of the lightest right-handed neutrino and sneutrino are equal. As in the case of the lepton decay asymmetries (13) the terms in the right-hand side of Eqs. (24) involving $\widetilde{A}_{j}$ stem from the self-energy diagrams while all other terms represent vertex corrections. Again the coefficients in front of $f^{S}(x)$ and $f^{V}(x)$ are not equal unlike the simplest realisations of Fukugita-Yanagida mechanism. From Eq. (24) it follows that the decay asymmetries induced by the additional decay modes (21) depend not only on the Yukawa couplings of exotic quarks and squarks to the right-handed neutrino but also on the couplings of the right-handed neutrino to leptons and sleptons. Extra CP asymmetries (24) tend to zero when the $Z_{2}^{H}$ symmetry violating Yukawa couplings $g_{k i j}^{N}$ vanish.

We can also define the overall decay asymmetries which are associated with each generation of exotic quarks, i.e.

$$
\varepsilon_{1, q_{k}}^{t o t}=\sum_{i} \varepsilon_{1, q_{k}}^{i}, \quad \quad \varepsilon_{\widetilde{1}, q_{k}}^{t o t}=\sum_{i} \varepsilon_{\widetilde{1}, q_{k}}^{i}
$$

The overall decay asymmetries that stem from the decays of the lightest right-handed neutrino and sneutrino can be presented in the following form

$$
\begin{gathered}
\varepsilon_{1, f}^{t o t}=\frac{1}{8 \pi\left(\operatorname{Tr} \Pi^{1}\right)} \sum_{j=2,3} \operatorname{Im}\left\{\widetilde{A}_{j} \Pi_{f f}^{j} f^{S}\left(\frac{M_{j}^{2}}{M_{1}^{2}}\right)+\left(\Pi^{j}\right)_{f f}^{2} f^{V}\left(\frac{M_{j}^{2}}{M_{1}^{2}}\right)\right\}, \\
\varepsilon_{1, k}^{t o t}=\frac{1}{8 \pi\left(\operatorname{Tr} \Omega^{1}\right)} \sum_{j=2,3} \operatorname{Im}\left\{\widetilde{A}_{j} \Omega_{k k}^{j} f^{S}\left(\frac{M_{j}^{2}}{M_{1}^{2}}\right)+\left(\Omega^{j}\right)_{k k}^{2} f^{V}\left(\frac{M_{j}^{2}}{M_{1}^{2}}\right)\right\}, \\
\widetilde{A}_{j}=\operatorname{Tr} \Pi^{j}+\frac{M_{1}}{M_{j}} \operatorname{Tr} \Pi^{j *}+\frac{3}{2}\left(\operatorname{Tr} \Omega^{j}+\frac{M_{1}}{M_{j}} \operatorname{Tr} \Omega^{j *}\right)
\end{gathered}
$$

where we set $\varepsilon_{1, D_{k}}^{t o t}=\varepsilon_{1, \widetilde{D}_{k}}^{t o t}=\varepsilon_{\widetilde{1}_{, D_{k}}}^{t o t}=\varepsilon_{\widetilde{1}_{,} \widetilde{D}_{k}}^{t o t}=\varepsilon_{1, k}^{t o t}, \Omega_{k i}^{j}=\sum_{m} g_{k m 1}^{N *} g_{i m j}^{N}$ while $\Pi_{m n}^{j}$ are given by Eqs. (16). Compact parametrisation of the overall CP asymmetries (26) allows elimination of a number of parameters on which total lepton asymmetry does not depend. 


\section{Numerical Results and Discussions}

We now consider the impact of new particles and interactions appearing in the $\mathrm{E}_{6} \mathrm{SSM}$ on the numerical values of the lepton $\mathrm{CP}$ asymmetries originating from the decays of the lightest right-handed neutrino and sneutrino. These decay asymmetries depend on all Yukawa couplings of neutrino superfields. Because the purpose of our studies here is to reveal the impact of extra couplings on the $\mathrm{CP}$ asymmetries we shall fix the Yukawa couplings of the lightest right-handed neutrino and sneutrino to lepton and Higgs superfields so that the observed pattern of neutrino masses and mixing angles is reproduced. Here, as an example, we concentrate on the see-saw models [8] with sequential dominance (SD) of right-handed neutrinos [32]-34] which lead to the appropriate neutrino spectrum in a technically natural way, i.e. small perturbations in the high energy input parameters do not change substantially the neutrino mass splittings at low energies. This means that small neutrino mass splittings are preserved in the presence of radiative correction 2 .

\subsection{Constrained Sequential Dominance}

To review how sequential dominance works we begin by writing the right-handed neutrino Majorana mass matrix in a diagonal basis as

$$
M_{R R}=\left(\begin{array}{ccc}
M_{1} & 0 & 0 \\
0 & M_{2} & 0 \\
0 & 0 & M_{3}
\end{array}\right)
$$

and the matrix of Yukawa couplings of the right-handed neutrino to lepton and Higgs fields $h_{i j}^{N}$ in terms of $(1,3)$ column vectors $A_{i}, B_{i}$ and $C_{i}$ as

$$
h_{i j}^{N}=\left(\begin{array}{lll}
A & B & C
\end{array}\right)=\left(\begin{array}{ccc}
d & a & a^{\prime} \\
e & b & b^{\prime} \\
f & c & c^{\prime}
\end{array}\right) .
$$

As before we assume that $M_{1} \ll M_{2} \ll M_{3}$. We shall also assume that $|d| \ll|e| \sim|f|$. The breakdown of electroweak symmetry induces Majorana mass terms for the lefthanded neutrino via the Yukawa interactions of the neutrino with the Higgs fields. After the integrating out the right-handed neutrino we get

$$
\mathcal{L}_{\text {mass }}^{\nu}=\frac{\left(\nu_{i}^{T} A_{i}\right)\left(A_{j}^{T} \nu_{j}\right)}{M_{1}} v_{2}^{2}+\frac{\left(\nu_{i}^{T} B_{i}\right)\left(B_{j}^{T} \nu_{j}\right)}{M_{2}} v_{2}^{2}+\frac{\left(\nu_{i}^{T} C_{i}\right)\left(C_{j}^{T} \nu_{j}\right)}{M_{3}} v_{2}^{2},
$$

\footnotetext{
${ }^{2}$ In general the radiative corrections in see-saw models may be sufficient to destroy (or create) the cancellations necessary to achieve the desired mass hierarchy [40].
} 
where $v_{2}$ is a VEV of the Higgs doublet $H_{u}$. The $3 \times 3$ mass matrix of the left-handed neutrino induced by $\mathcal{L}_{\text {mass }}^{\nu}$ can be diagonalised by means of a unitary transformation that may be written as a sequence of transformations

$$
V^{\nu \dagger}=P R_{23} U_{13} R_{12} P_{12}
$$

where

$$
\begin{aligned}
& P=\left(\begin{array}{ccc}
e^{i \omega_{1}} & 0 & 0 \\
0 & e^{i \omega_{2}} & 0 \\
0 & 0 & e^{i \omega_{3}}
\end{array}\right), \quad R_{23}=\left(\begin{array}{ccc}
1 & 0 & 0 \\
0 & c_{23}^{\nu} & s_{23}^{\nu} \\
0 & -s_{23}^{\nu} & c_{23}^{\nu} \\
c_{13}^{\nu} & 0 & s_{13}^{\nu} e^{-i \delta^{\nu}} \\
0 & 1 & 0 \\
-s_{13}^{\nu} e^{i \delta^{\nu}} & 0 & c_{13}^{\nu}
\end{array}\right), \quad R_{12}=\left(\begin{array}{ccc}
c_{12}^{\nu} & s_{12}^{\nu} & 0 \\
-s_{12}^{\nu} & c_{12}^{\nu} & 0 \\
0 & 0 & 1
\end{array}\right), \\
& P_{12}=\left(\begin{array}{ccc}
e^{i \beta_{1}} & 0 & 0 \\
0 & e^{i \beta_{2}} & 0 \\
0 & 0 & 1
\end{array}\right) \text {, }
\end{aligned}
$$

and $s_{i j}^{\nu}=\sin \theta_{i j}^{\nu}, c_{i j}^{\nu}=\cos \theta_{i j}^{\nu}$. The phase matrix $P$ in the right hand side of Eq. (30) may always be removed by an additional charged lepton phase rotation.

The Pontecorvo-Maki-Nakagawa-Sakata (PMNS) neutrino mixing matrix $U_{P M N S}$ [4] is a product of unitary matrices $V^{E}$ and $V^{\nu \dagger}$, where $V^{E}$ is associated with the diagonalisation of the charged lepton mass matrix. Since the charged lepton mixing angles are expected to be small $U_{P M N S} \approx V^{\nu \dagger}$ in the first approximation. The only exception is $\theta_{13}$. The CHOOZ experiment sets a stringent constraint on the value of $\theta_{13} \lesssim 0.2$ [42]. Because $\theta_{13}$ is small it receives important contributions not just from $\theta_{13}^{\nu}$, but also from the charged lepton angles [33]. Further we will assume that $\theta_{13}^{\nu} \ll 1$.

The sequential dominance implies that the first term in Eq. (29) gives a dominant contribution to the mass matrix of the left-handed neutrino, the second term is subdominant whereas the contribution of the last term in Eq. (29) is negligible [32]-34]. This structure of the mass terms guarantees that the mass of the heaviest left-handed neutrino $m_{3}$ is much larger than the mass of the second lightest one. If the heaviest left-handed neutrino is denoted $\nu_{3}$ then sequential dominance results in the physical neutrino eigenstate $\nu_{3} \simeq d \nu_{e}+e \nu_{\mu}+f \nu_{\tau}$ with the mass 33 ]

$$
\left|m_{3}\right| \simeq\left(|d|^{2}+|e|^{2}+|f|^{2}\right) v_{2}^{2} / M_{1}
$$

Two other orthogonal combinations of neutrinos remain massless in the leading approximation. The requirement of a small angle $\theta_{13}^{\nu}$ implies that $|d| \ll|e|,|f|$. Then the atmospheric angle $\theta_{23}$ is given by [33]

$$
\tan \theta_{23} \approx \tan \theta_{23}^{\nu} \approx \frac{|e|}{|f|} .
$$


Although the leading approximation allows us to get an appropriate description of atmospheric neutrino data, we need to go beyond it to account for the data of other neutrino experiments. The contribution of the sub-leading right-handed neutrino does not substantially change the mass of the heaviest left-handed neutrino state (32) and atmospheric angle (33). However it gives rise to non-zero second lightest neutrino mass. The sub-leading contributions to the left-handed neutrino mass matrix also induce mixing between the heaviest and other left-handed neutrino states. The neutrino mass matrix can be reduced to the block diagonal form by means of unitary transformations $U_{13}$ if

$$
\theta_{13}^{\nu} \approx e^{i\left(\tilde{\phi}+\phi_{a}-\phi_{e}\right)} \frac{|a|\left(e^{*} b+f^{*} c\right)}{\left(|e|^{2}+|f|^{2}\right)^{3 / 2}} \frac{M_{1}}{M_{2}}+e^{i\left(\tilde{\phi}+\phi_{d}-\phi_{e}\right)} \frac{|d|}{\sqrt{|e|^{2}+|f|^{2}}},
$$

where $\phi_{x}$ are the phases of Yukawa couplings, i.e. $x=|x| e^{i \phi_{x}}$. The relative phase $\phi_{e}-\phi_{f}$ is chosen so that the angle $\theta_{23}^{\nu}$ is real. The phase $\tilde{\phi}$ is fixed by the requirement that the angle $\theta_{13}$ is real and positive. When $d=0$ we get

$$
\tilde{\phi}=\phi_{e}-\phi_{a}-\zeta, \quad \zeta=\arg \left(e^{*} b+f^{*} c\right) .
$$

It is worth to notice here that the angle $\theta_{13}^{\nu}$ is automatically small in the considered approximation.

Finally, the left-handed neutrino mass matrix can be completely diagonalised by the $R_{12}$ rotation. Then the second lightest left-handed neutrino gets mass [33]

$$
\left|m_{2}\right| \simeq \frac{|a|^{2} v_{2}^{2}}{M_{2} \sin ^{2} \theta_{12}^{\nu}}
$$

while the solar angle is given by 33

$$
\begin{gathered}
\tan \theta_{12} \approx \tan \theta_{12}^{\nu} \simeq \frac{a}{b \cos \theta_{23}-c e^{i\left(\phi_{e}-\phi_{f}\right)} \sin \theta_{23}}=\frac{|a|}{|b| c_{23} \cos \phi_{b}^{\prime}-|c| s_{23} \cos \phi_{c}^{\prime}}, \\
\phi_{b}^{\prime}=\phi_{b}-\phi_{a}-\tilde{\phi}-\delta, \quad \phi_{c}^{\prime}=\phi_{c}-\phi_{a}+\phi_{e}-\phi_{f}-\tilde{\phi}-\delta .
\end{gathered}
$$

Once again the phases can be chosen so that $\tan \theta_{12}^{\nu}$ is real and positive. This can be achieved if phases $\phi_{b}^{\prime}$ and $\phi_{c}^{\prime}$ satisfy the condition

$$
|b| c_{23} \sin \phi_{b}^{\prime} \approx|c| s_{23} \sin \phi_{c}^{\prime}
$$

Note that in contrast with $\theta_{13}^{\nu}$ the solar angle (37) is completely determined by the subleading couplings due to a natural cancellation of the leading contributions. Therefore this angle should be relatively large. The lightest left-handed neutrino state remains massless in the considered approximation. Its mass is generated by the sub-sub-leading couplings of the heaviest right-handed neutrino, i.e.

$$
\left|m_{1}\right| \simeq O\left(\frac{|C|^{2} v_{2}^{2}}{M_{3}}\right)
$$


Thus sequential dominance results in a full neutrino mass hierarchy $m_{1} \ll m_{2} \ll m_{3}$. Because SD does not require any fine tuning the contribution of radiative corrections to the neutrino masses and mixing angles is expected to be quite small, at the level of a few per cent [43].

Current neutrino oscillation data point strongly to a specific form for the lepton mixing matrix with effective bimaximal mixing of $\nu_{\mu}$ and $\nu_{\tau}$ at the atmospheric scale and effective trimaximal mixing for $\nu_{e}, \nu_{\mu}$ and $\nu_{\tau}$ at solar scale (tri-bimaximal mixing [44]). In the tri-bimaximal mixing scenario the PMNS matrix takes a form

$$
U_{P M N S} \simeq\left(\begin{array}{ccc}
\sqrt{\frac{2}{3}} & \sqrt{\frac{1}{3}} & 0 \\
-\sqrt{\frac{1}{6}} & \sqrt{\frac{1}{3}} & \sqrt{\frac{1}{2}} \\
\sqrt{\frac{1}{6}} & -\sqrt{\frac{1}{3}} & \sqrt{\frac{1}{2}}
\end{array}\right) .
$$

Comparing matrix (40) with the general parametrisation of the neutrino mixing matrix (30) one can easily establish that tri-bimaximal mixing scenario corresponds to $\theta_{13}=0$, $\sin \theta_{12}=1 / \sqrt{3}$ and $\theta_{23}=\pi / 4$. Within the framework of sequential dominance the vanishing of the mixing angle $\theta_{13}$ can be naturally achieved when

$$
d \simeq 0, \quad e^{*} b+f^{*} c=\left(A^{\dagger} B\right) \simeq 0 .
$$

Since in this case the bimaximal mixing between $\nu_{\mu}$ and $\nu_{\tau}$ implies that $|e|=|f|$ the conditions (41) constrain the Yukawa couplings of the second lightest right-handed neutrino. In particular, from Eq. (41) it follows that $|b|=|c|$. Taking into account that tri-bimaximal mixing also requires $\sin \theta_{12}=1 / \sqrt{3}$ one can show that within the sequential dominance the Yukawa couplings of the lightest and second lightest right-handed neutrinos which correspond to the tri-bimaximal mixing scenario can be always chosen so that

$$
d \simeq 0, \quad f=-e=|A| e^{i \phi_{A}}, \quad a=b=c=|B| e^{i \phi_{B}} .
$$

This is so-called constrained sequential dominance (CSD) [45]. Note that CSD does not constrain the Yukawa couplings of the heaviest right-handed neutrino $a^{\prime}, b^{\prime}$ and $c^{\prime}$ because they only give sub-sub-dominant contribution to the neutrino mass matrix. Different issues concerning the leptogenesis in the neutrino models based on the seesaw mechanism and sequential right-handed neutrino dominance were discussed in [34, 15].

\subsection{Results of numerical analysis}

\subsection{1 $\quad \mathrm{E}_{6} \mathrm{SSM}$ with unbroken $Z_{H}^{2}$ symmetry}

With the assumption of the constrained sequential dominance we calculate the values of the decay asymmetries in the $\mathrm{E}_{6} \mathrm{SSM}$. According to CSD one can ignore the contribu- 
tion of the heaviest right-handed neutrino so that the analytical expressions for the CP asymmetries derived in Section 3 are considerably simplified. We start our analysis from the $\mathrm{E}_{6} \mathrm{SSM}$ with exact $Z_{H}^{2}$ symmetry. In this case there is only one extra $\mathrm{CP}$ asymmetry associated with the decay of the lightest right-handed neutrino into scalar (fermion) components of the fourth lepton doublet superfield $L_{4}$ and Higgsinos (Higgs bosons). Substituting the pattern of Yukawa couplings that corresponds to the constrained sequential dominance into Eqs. (18) and neglecting the contribution of the heaviest right-handed neutrino to the $\mathrm{CP}$ asymmetries we get

$$
\begin{aligned}
\varepsilon_{1, L_{4}}^{3} & \simeq \frac{3}{8 \pi} \frac{\left|h_{H_{3}^{u} L_{4} N_{1}}^{N}\right|^{2}\left|h_{H_{3}^{u} L_{4} N_{2}}^{N}\right|^{2} \sin \phi_{L}}{2|A|^{2}+\left|h_{H_{3}^{u} L_{4} N_{1}}^{N}\right|^{2}} \frac{M_{1}}{M_{2}}, \quad \varepsilon_{1, e}^{3}=0, \\
\varepsilon_{1, \tau}^{3} & \simeq-\varepsilon_{1, \mu}^{3} \simeq \frac{3}{8 \pi} \frac{\left|h_{H_{3}^{u} L_{4} N_{1}}^{N}\right| h_{H_{3}^{u} L_{4} N_{2}}^{N}|| A|| B \mid \sin \phi_{\mu \tau}}{2|A|^{2}+\left|h_{H_{3}^{u} L_{4} N_{1}}^{N}\right|^{2}} \frac{M_{1}}{M_{2}}, \\
\phi_{\mu \tau} & =\phi_{41}+\phi_{A}-\phi_{42}-\phi_{B}, \quad \phi_{L}=2\left(\phi_{41}-\phi_{42}\right),
\end{aligned}
$$

where $h_{H_{3}^{u} L_{4} N_{1}}^{N} \equiv h_{341}^{N}=h_{41}^{N}, h_{H_{3}^{u} L_{4} N_{2}}^{N} \equiv h_{342}^{N}=h_{42}^{N}, h_{H_{3}^{u} L_{4} N_{1}}^{N}=\left|h_{H_{3}^{u} L_{4} N_{1}}^{N}\right| e^{i \phi_{41}}$ and $h_{H_{3}^{u} L_{4} N_{2}}^{N}=\left|h_{H_{3}^{u} L_{4} N_{2}}^{N}\right| e^{i \phi_{42}}$. Note that in the limit when $h_{H_{3}^{u} L_{4} N_{1}}^{N}$ and $h_{H_{3}^{u} L_{4} N_{2}}^{N}$ go to zero all $\mathrm{CP}$ asymmetries vanish. This is not an accident. When Yukawa couplings $h_{H_{3}^{u} L_{4} N_{1}}^{N}$ and $h_{H_{3}^{u} L_{4} N_{2}}^{N}$ tend to zero the interactions of the right-handed neutrinos with the Higgs and lepton superfields are exactly the same as in the MSSM. At the same time the conditions (41) which result in the natural realisation of the tri-bimaximal mixing scenario in the framework of sequential dominance ensure the vanishing of all decay asymmetries within the SM and the MSSM. Thus the induced values of the lepton decay asymmetries (43) are entirely caused by the new particles and interactions appearing in the $\mathrm{E}_{6} \mathrm{SSM}$.

The CP asymmetries (43) also vanish when all Yukawa couplings are real, i.e. CP invariance in the lepton sector is preserved. The decay asymmetries $\varepsilon_{1, L_{4}}^{3}$ and $\varepsilon_{1, \tau}^{3}=-\varepsilon_{1, \mu}^{3}$ attain their maximum absolute values when $\sin \phi_{L}$ and $\sin \phi_{\mu \tau}$ are equal to \pm 1 respectively. The maximum absolute values of the $\mathrm{CP}$ asymmetries (43) are given by

$$
\begin{aligned}
\left|\varepsilon_{1, L_{4}}^{3}\right| & \simeq \frac{3}{8 \pi} \frac{\left|h_{H_{3}^{u} L_{4} N_{1}}^{N}\right|^{2}\left|h_{H_{3}^{u} L_{4} N_{2}}^{N}\right|^{2}}{2|A|^{2}+\left|h_{H_{3}^{u} L_{4} N_{1}}^{N}\right|^{2}} \frac{M_{1}}{M_{2}}, \\
\left|\varepsilon_{1, \tau}^{3}\right| & =\left|\varepsilon_{1, \mu}^{3}\right| \simeq \frac{3}{8 \pi} \frac{\left|h_{H_{3}^{u} L_{4} N_{1}}^{N}\right| h_{H_{3}^{u} L_{4} N_{2}}^{N}|| A|| B \mid}{2|A|^{2}+\left|h_{H_{3}^{u} L_{4} N_{1}}^{N}\right|^{2}} \frac{M_{1}}{M_{2}} .
\end{aligned}
$$

The dependence of the maximum values of $\left|\varepsilon_{1, L_{4}}^{3}\right|$ and $\left|\varepsilon_{1, \tau}^{3}\right|=\left|\varepsilon_{1, \mu}^{3}\right|$ on the absolute values of the additional Yukawa couplings $\left|h_{H_{3}^{u} L_{4} N_{1}}^{N}\right|$ and $\left|h_{H_{3}^{u} L_{4} N_{2}}^{N}\right|$ is examined in Fig. 4 where we fix $\left(M_{2} / M_{1}\right)=10$. To avoid problems related with the overproduction of gravitinos we assume that the mass of the lightest right-handed neutrino is relatively small $M_{1} \simeq 10^{6} \mathrm{GeV}$. We also set $v_{2}=v \simeq 246 \mathrm{GeV}$ that corresponds to large values of $\tan \beta$ and choose parameters $|A|$ and $|B|$ so that the observed neutrino mass-squared differences are reproduced (see, for example, [46]). 
In Figs. $4 \mathrm{a}$ and $4 \mathrm{~b}$ the dependence of the maximum value of $\left|\varepsilon_{1, \tau}^{3}\right|=\left|\varepsilon_{1, \mu}^{3}\right|$ on $\left|h_{H_{3}^{u} L_{4} N_{1}}^{N}\right|$ and $\left|h_{H_{3}^{u} L_{4} N_{2}}^{N}\right|$ is studied whereas in Figs. $4 \mathrm{c}$ and $4 \mathrm{~d}$ we plot the maximum value of $\left|\varepsilon_{1, L_{4}}^{3}\right|$ as a function of new Yukawa couplings. From Eqs. (44) and Figs. 4a and 4c it follows that both maximum absolute values of the CP asymmetries (44) grow monotonically with increasing of $\left|h_{H_{3}^{u} L_{4} N_{2}}^{N}\right|$. The dependence of $\left|\varepsilon_{1, L_{4}}^{3}\right|$ and $\left|\varepsilon_{1, \tau}^{3}\right|=\left|\varepsilon_{1, \mu}^{3}\right|$ on $\left|h_{H_{3}^{u} L_{4} N_{1}}^{N}\right|$ is more complicated. At small values of $\left|h_{H_{3}^{u} L_{4} N_{1}}^{N}\right|$ these decay asymmetries are small and increase when $\left|h_{H_{3}^{u} L_{4} N_{1}}^{N}\right|$ becomes larger. However if $\left|h_{H_{3}^{u} L_{4} N_{1}}^{N}\right|$ is much larger than $|A|$ the maximum absolute values of $\left|\varepsilon_{1, \tau}^{3}\right|=\left|\varepsilon_{1, \mu}^{3}\right|$ is inversely proportional to $\left|h_{H_{3}^{u} L_{4} N_{1}}^{N}\right|$ and therefore diminishes with increasing of $\left|h_{H_{3}^{u} L_{4} N_{1}}^{N}\right|$ while $\left|\varepsilon_{1, L_{4}}^{3}\right|$ reaches its saturation limit (see Figs. 4b and $4 \mathrm{~d}$ ). The CP asymmetries $\left|\varepsilon_{1, \tau}^{3}\right|=\left|\varepsilon_{1, \mu}^{3}\right|$ attain their maximal possible value at $\left|h_{H_{3}^{u} L_{4} N_{1}}^{N}\right| \simeq \sqrt{2}|A|$. Thus we establish the following theoretical restrictions on the values of decay asymmetries

$$
\left|\varepsilon_{1, L_{4}}^{3}\right| \lesssim \frac{3 M_{1}}{8 \pi M_{2}}\left|h_{H_{3}^{u} L_{4} N_{2}}^{N}\right|^{2}, \quad \quad\left|\varepsilon_{1, \tau}^{3}\right|=\left|\varepsilon_{1, \mu}^{3}\right| \lesssim \frac{3 \sqrt{2} M_{1}}{32 \pi M_{2}}\left|h_{H_{3}^{u} L_{4} N_{2}}^{N}\right||B| .
$$

One can easily see that the theoretical upper bounds on the absolute values of the $\mathrm{CP}$ asymmetries (45) are determined by the Yukawa couplings of the second lightest righthanded neutrino and do not depend on the Yukawa couplings of the lightest right-handed neutrino. In general the maximal absolute values of decay asymmetries diminish when the couplings $\left|h_{H_{3}^{u} L_{4} N_{1}}^{N}\right|$ and $\left|h_{H_{3}^{u} L_{4} N_{2}}^{N}\right|$ decrease (see Fig. 5).

There is also another general tendency that should be mentioned here. When $M_{1} \ll 10^{13}-10^{14} \mathrm{GeV}$ the absolute value of the CP asymmetry associated with the decay $N_{1} \rightarrow L_{4}+H_{u}$ tend to be considerably larger than lepton decay asymmetries $\varepsilon_{1, \mu}^{3}$ and $\varepsilon_{1, \tau}^{3}$ (see Figs. 4-5). This happens because lower masses of the right-handed neutrinos require smaller values of the Yukawa couplings of the Higgs doublet $H_{u}$ to leptons. Otherwise the observed neutrino mass-squared differences can not be reproduced within the framework of sequential dominance. From Eqs. (32) and (36) it follows that $|A| \propto \sqrt{M_{1}\left|m_{3}\right| / v^{2}}$ while $|B| \propto \sqrt{M_{2}\left|m_{2}\right| / v^{2}}$. Thus for a fixed ratio $M_{1} / M_{2}$ the maximal possible values of the decay asymmetries $\left|\varepsilon_{1, \mu}^{3}\right|$ and $\left|\varepsilon_{1, \tau}^{3}\right|$ (45) diminishes as $\sqrt{M_{1}}$ when $M_{1}$ decreases. In fact, the decrease of lepton CP asymmetries with the mass of the lightest right-handed neutrino is a common feature of most see-saw models. This results in the lower bound on the lightest right-handed neutrino mass: $M_{1} \gtrsim 10^{9} \mathrm{GeV}$ [19]. At the same time the results of our analysis presented in Figs. 5 demonstrate that within the $\mathrm{E}_{6} \mathrm{SSM}$ with unbroken $Z_{2}^{H}$ it is possible to generate an appreciable value of the $\mathrm{CP}$ asymmetry $\left|\varepsilon_{1, L_{4}}^{3}\right|=10^{-6}-10^{-4}$ even for $M_{1}=10^{6} \mathrm{GeV}$. This can be achieved if the Yukawa couplings of the fourth lepton doublet $L_{4}$ to the Higgs fields $H_{u}$ vary from 0.01 to 0.1. At low energies the induced lepton asymmetry is transferred to the ordinary lepton asymmetries via the decays of heavy $L_{4}$ and $\tilde{L}_{4}$ into leptons (sleptons) and Higgs fields 
$H_{d}\left(\operatorname{Higgsinos} \tilde{H}_{d}\right)$.

\subsection{2 $\quad \mathrm{E}_{6} \mathrm{SSM}$ Model I}

In the case of the $\mathrm{E}_{6} \mathrm{SSM}$ Model I two generations of inert-Higgs superfields $H_{\alpha}^{u}(\alpha=1,2)$ contribute to $\varepsilon_{1, \ell_{x}}$ through loop diagrams and give rise to a set of extra decay asymmetries $\varepsilon_{1, \ell_{x}}^{\alpha}$ defined by Eq. (12). Because the Yukawa couplings of $H_{\alpha}^{u}$ to the quarks and leptons of the first two generation are expected to be rather small in order to avoid non-diagonal flavour transitions we assume that inert Higgs fields couple to the third generation fermions only. To simplify our analysis further we also assume that only one inert Higgs doublet $H_{2}^{u}$ has non-zero couplings with the doublet of leptons of the third generation and right-handed neutrinos. Then the analytic expression (15) for the overall $\mathrm{CP}$ asymmetries reduces to

$$
\begin{aligned}
& \varepsilon_{1, \mu}^{t o t} \simeq \frac{1}{4 \pi} \frac{\left|h_{H_{2}^{u} L_{3} N_{1}}^{N}\right|\left|h_{H_{2}^{u} L_{3} N_{2}}^{N}\right||A||B| \sin \phi_{\mu}}{2|A|^{2}+\left|h_{H_{2}^{u} L_{3} N_{1}}\right|^{2}} \frac{M_{1}}{M_{2}}, \quad \varepsilon_{1, e}^{t o t}=0, \\
& \varepsilon_{1, \tau}^{t o t} \simeq \frac{\left(4\left|h_{H_{2}^{u} L_{3} N_{1}}^{N}\right|\left|h_{H_{2}^{u} L_{3} N_{2}}^{N}\right||A||B| \sin \phi_{\mu}+3\left|h_{H_{2}^{u} L_{3} N_{1}}^{N}\right|^{2}\left|h_{H_{2}^{u} L_{3} N_{2}}^{N}\right|^{2} \sin \phi_{\tau}\right)}{8 \pi\left(2|A|^{2}+\left|h_{H_{2}^{u} L_{3} N_{1}}^{N}\right|^{2}\right)} \frac{M_{1}}{M_{2}}, \\
& \phi_{\tau}=2\left(\phi_{231}-\phi_{232}\right),
\end{aligned}
$$

where $h_{H_{2}^{u} L_{3} N_{1}}^{N} \equiv h_{231}^{N}, \quad h_{H_{2}^{u} L_{3} N_{2}}^{N} \equiv h_{232}^{N}, \quad h_{H_{2}^{u} L_{3} N_{1}}^{N}=\left|h_{H_{2}^{u} L_{3} N_{1}}^{N}\right| e^{i \phi_{231}}$ and $h_{H_{2}^{u} L_{3} N_{2}}^{N}=\left|h_{H_{2}^{u} L_{3} N_{2}}^{N}\right| e^{i \phi_{232}}$. Here, to clarify the contribution of the inert-Higgs doublet, we set all Yukawa couplings of $L_{4}$ to the right-handed neutrinos to be zero.

As before the overall CP asymmetries (46) vanish in the MSSM limit of the $\mathrm{E}_{6} \mathrm{SSM}$ when $h_{H_{2}^{u} L_{3} N_{1}}^{N}$ and $h_{H_{2}^{u} L_{3} N_{2}}^{N}$ go to zero. The decay asymmetries (46) also tend to zero if $\mathrm{CP}$ invariance is preserved in the lepton sector, i.e. phases of all Yukawa couplings vanish. Once again $\varepsilon_{1, \mu}^{t o t}$ and $\varepsilon_{1, \tau}^{t o t}$ reach their maximum absolute values when $\sin \phi_{\mu}$ and $\sin \phi_{\tau}$ are equal to \pm 1 . The corresponding maximum absolute values of the overall $\mathrm{CP}$ asymmetries (46)) can be written as

$$
\begin{aligned}
\left|\varepsilon_{1, \mu}^{t o t}\right| & \simeq \frac{1}{4 \pi} \frac{\left|h_{H_{2}^{u} L_{3} N_{1}}^{N}\right|\left|h_{H_{2}^{u} L_{3} N_{2}}^{N}\right||A||B|}{2|A|^{2}+\left|h_{H_{2}^{u} L_{3} N_{1}}^{N}\right|^{2}} \frac{M_{1}}{M_{2}} \\
\left|\varepsilon_{1, \tau}^{t o t}\right| & \simeq \frac{\left(4\left|h_{H_{2}^{u} L_{3} N_{1}}^{N}\right|\left|h_{H_{2}^{u} L_{3} N_{2}}^{N}\right||A||B|+3\left|h_{H_{2}^{u} L_{3} N_{1}}^{N}\right|^{2}\left|h_{H_{2}^{u} L_{3} N_{2}}^{N}\right|^{2}\right)}{8 \pi\left(2|A|^{2}+\left|h_{H_{2}^{u} L_{3} N_{1}}^{N}\right|^{2}\right)} \frac{M_{1}}{M_{2}} .
\end{aligned}
$$

In Figs. 6-7 we present the results of our numerical analysis of the decay asymmetries in the $\mathrm{E}_{6} \mathrm{SSM}$ Model I. The dependence of the maximum values of $\left|\varepsilon_{1, \mu}^{t o t}\right|$ and $\left|\varepsilon_{1, \tau}^{t o t}\right|$ on $\left|h_{H_{2}^{u} L_{3} N_{1}}^{N}\right|$ and $\left|h_{H_{2}^{u} L_{3} N_{2}}^{N}\right|$ is studied in Fig. 6. As before we set $\left(M_{2} / M_{1}\right)=10$, $M_{1} \simeq 10^{6} \mathrm{GeV}, v_{2} \simeq v \simeq 246 \mathrm{GeV}$ and adjust parameters $|A|$ and $|B|$ to reproduce the observed neutrino mass-squared differences. In Figs. $6 \mathrm{a}$ and $6 \mathrm{~b}$ we plot the maximum value 
of $\left|\varepsilon_{1, \mu}^{\text {tot }}\right|$ as a function of $\left|h_{H_{2}^{u} L_{3} N_{1}}^{N}\right|$ and $\left|h_{H_{2}^{u} L_{3} N_{2}}^{N}\right|$ while the dependence of the maximum value of $\left|\varepsilon_{1, \tau}^{t o t}\right|$ on these new Yukawa couplings is explored in Figs. 6c and 6d. From Eq. (47) one can see that at very small values of new Yukawa couplings $\left(\left|h_{H_{2}^{u} L_{3} N_{1}}^{N}\right|,\left|h_{H_{2}^{u} L_{3} N_{2}}^{N}\right| \ll|A|\right.$ and $|B|$ ) the maximum absolute values of the overall CP asymmetry are proportional to $\left|h_{H_{2}^{u} L_{3} N_{1}}^{N}\right| \cdot\left|h_{H_{2}^{u} L_{3} N_{2}}^{N}\right|$. At so small values of $\left|h_{H_{2}^{u} L_{3} N_{1}}^{N}\right|$ and $\left|h_{H_{2}^{u} L_{3} N_{2}}^{N}\right|$ the maximum absolute value of the overall $\mathrm{CP}$ asymmetry associated with the decay of $N_{1}$ into $\tau$-lepton is twice larger than the maximum value of $\left|\varepsilon_{1, \mu}^{t o t}\right|$. The maximum values of $\left|\varepsilon_{1, \mu}^{t o t}\right|$ and $\left|\varepsilon_{1, \tau}^{t o t}\right|$ rise with increasing of $\left|h_{H_{2}^{u} L_{3} N_{2}}^{N}\right|$ (see Fig. 6a and 6c). When $\left|h_{H_{2}^{u} L_{3} N_{2}}^{N}\right| \gg|A|,|B|$ the value of $\left|\varepsilon_{1, \tau}^{t o t}\right|$ tends to be much larger than $\left|\varepsilon_{1, \mu}^{\text {tot }}\right|$.

At small values of $\left|h_{H_{2}^{u} L_{3} N_{1}}^{N}\right|$ the maximum absolute values of both decay asymmetries also grow with increasing of $\left|h_{H_{2}^{u} L_{3} N_{1}}^{N}\right|$ independently of $\left|h_{H_{2}^{u} L_{3} N_{2}}^{N}\right|$ (see Fig. 6b and 6d). But $\left|\varepsilon_{1, \mu}^{\text {tot }}\right|$ attains its maximum possible value at $\left|h_{H_{2}^{u} L_{3} N_{1}}^{N}\right|=\sqrt{2}|A|$ whereas $\left|\varepsilon_{1, \tau}^{\text {tot }}\right|$ approach its upper bound at large values of $\left|h_{H_{2}^{u} L_{3} N_{1}}^{N}\right| \gg|A|,|B|$. When $\left|h_{H_{2}^{u} L_{3} N_{1}}^{N}\right|$ is significantly larger than $|A|$ and $|B|$ the the maximum value of $\left|\varepsilon_{1, \mu}^{t}\right|$ is inversely proportional to $\left|h_{H_{2}^{u} L_{3} N_{1}}^{N}\right|$ while $\left|\varepsilon_{1, \tau}^{\text {tot }}\right|$ is almost independent of $\left|h_{H_{2}^{u} L_{3} N_{1}}^{N}\right|$. In the considered case the theoretical upper bounds on $\left|\varepsilon_{1, \mu}^{t o t}\right|$ and $\left|\varepsilon_{1, \tau}^{t o t}\right|$ are given by

$$
\begin{aligned}
\left|\varepsilon_{1, \tau}^{t o t}\right| & \lesssim \frac{M_{1}}{8 \pi M_{2}}\left|h_{H_{2}^{u} L_{3} N_{2}}^{N}\right|^{2}\left[3+\frac{4 x}{12+\sqrt{8 x+9}}\right], \quad x=\frac{|B|^{2}}{\left|h_{H_{2}^{u} L_{3} N_{2}}^{N}\right|^{2}}, \\
\left|\varepsilon_{1, \mu}^{t o t}\right| & \lesssim \frac{\sqrt{2} M_{1}}{16 \pi M_{2}}\left|h_{H_{2}^{u} L_{3} N_{2}}^{N}\right||B| .
\end{aligned}
$$

As before the theoretical restrictions on the absolute values of CP asymmetries (48) are set by the Yukawa couplings of the second lightest right-handed neutrino and independent of the Yukawa couplings of the lightest right-handed neutrino. Because for a fixed ratio $M_{1} / M_{2}$ the values of $|A|$ and $|B| \propto \sqrt{M_{1}}$ the maximum possible value of $\left|\varepsilon_{1, \mu}^{\text {tot }}\right|$ decreases when $M_{1}$ becomes smaller while the theoretical upper bound on $\left|\varepsilon_{1, \tau}^{\text {tot }}\right|$ does not change much. As a consequence $\left|\varepsilon_{1, \tau}^{\text {tot }}\right|$ tends to dominate over $\left|\varepsilon_{1, \mu}^{t o t}\right|$ at low masses of the lightest right-handed neutrino $M_{1} \ll 10^{13}-10^{14} \mathrm{GeV}$ (see Figs. 6-7). Since the maximum possible value of $\left|\varepsilon_{1, \tau}^{t o t}\right|$ is determined mainly by $\left|h_{H_{2}^{u} L_{3} N_{2}}^{N}\right|$, which is not constrained by the neutrino oscillation data, an appreciable CP asymmetry within the $\mathrm{E}_{6} \mathrm{SSM}$ Model I can be induced even when $M_{1}$ is relatively low. Fig. 7 demonstrates that for $M_{1} \simeq 10^{6} \mathrm{GeV}$ the decay asymmetry $\left|\varepsilon_{1, \tau}^{t o t}\right|=10^{-6}-10^{-4}$ can be generated if $\left|h_{H_{2}^{u} L_{3} N_{2}}^{N}\right|$ varies from 0.01 to 0.1 .

\subsection{3 $\mathrm{E}_{6} \mathrm{SSM}$ Model II}

Within the $\mathrm{E}_{6} \mathrm{SSM}$ Model II the lightest right-handed neutrino may decay into the leptoquarks (squarks) and down-type squarks (down-type quarks). New decay modes of the lightest right-handed neutrino lead to the set of extra CP asymmetries $\varepsilon_{1, D_{k}}^{i}$ (23) which appear in addition to those arising in the $\mathrm{E}_{6} \mathrm{SSM}$ Model I. Leptoquarks also give a substan- 
tial contribution to $\varepsilon_{1, \ell_{x}}^{k}$, through loop diagrams if the corresponding Yukawa couplings $g_{k i j}^{N}$ are large enough. By construction the exotic quarks and squarks in the $\mathrm{E}_{6} \mathrm{SSM}$ couple predominantly to the the quark and lepton superfields of the third generation. Therefore in our analysis we neglect the Yukawa couplings of the exotic quarks and squarks to the first and second generation particles. Moreover for simplicity we assume that only the third generation exotic quarks and squarks have appreciable couplings to the bosons and fermions of the third generation and the Yukawa couplings of $L_{4}$ and $H_{\alpha}^{u}$ to the righthanded neutrinos vanish. In this approximation for the maximum absolute values of the CP asymmetries $\left|\varepsilon_{1, \tau}^{3}\right|=\left|\varepsilon_{1, \mu}^{3}\right|$ and $\left|\varepsilon_{1, D_{3}}^{3}\right|$ one obtains

$$
\left|\varepsilon_{1, \tau}^{3}\right|=\left|\varepsilon_{1, \mu}^{3}\right| \simeq \frac{3|B| M_{1}}{16 \pi|A| M_{2}}\left|g_{D_{3} d_{3} N_{1}}^{N}\right|\left|g_{D_{3} d_{3} N_{2}}^{N}\right|, \quad\left|\varepsilon_{1, D_{3}}^{3}\right| \simeq \frac{3 M_{1}}{2 \pi M_{2}}\left|g_{D_{3} d_{3} N_{2}}^{N}\right|^{2},(
$$

where $g_{D_{3} d_{3} N_{1}}^{N} \equiv g_{331}^{N}$ and $g_{D_{3} d_{3} N_{2}}^{N}=g_{332}^{N}$. All other decay asymmetries vanish in the considered approximation. As before the maximum absolute values of the $\mathrm{CP}$ asymmetries (49) tend to zero if $g_{D_{3} d_{3} N_{1}}^{N} \rightarrow 0$ and $g_{D_{3} d_{3} N_{2}}^{N} \rightarrow 0$. However in contrast with the scenarios considered before the absolute values of the CP asymmetries $\left|\varepsilon_{1, \mu}^{3}\right|$ and $\left|\varepsilon_{1, \tau}^{3}\right|$ do not change when the lightest right-handed neutrino mass varies while $M_{1} / M_{2}$ remains intact. Indeed, according to the Eqs. (32) and (36) the ratio $|A| /|B|$ is proportional to $\sqrt{M_{1} / M_{2}}$. As a result the explicit dependence of the lepton decay asymmetries on the right-handed neutrino mass scale in Eq. (49) is cancelled. The maximum absolute values of the CP asymmetries (49) are determined by $\left|g_{D_{3} d_{3} N_{1}}^{N}\right|$ and $\left|g_{D_{3} d_{3} N_{2}}^{N}\right|$.

The dependence of the maximum values of $\left|\varepsilon_{1, \tau}^{3}\right|=\left|\varepsilon_{1, \mu}^{3}\right|$ and $\left|\varepsilon_{1, D_{3}}^{3}\right|$ on the Yukawa couplings $g_{D_{3} d_{3} N_{1}}^{N}$ and $g_{D_{3} d_{3} N_{2}}^{N}$ is examined in Fig. 8. Once again we fix $\left(M_{2} / M_{1}\right)=10$, $v_{2} \simeq 246 \mathrm{GeV}$ and choose $|A|$ and $|B|$ so that the phenomenologically acceptable pattern of the neutrino mass spectrum is reproduced. From Eq. (49) and Fig. 8 one can see that the decay asymmetries $\left|\varepsilon_{1, \tau}^{3}\right|=\left|\varepsilon_{1, \mu}^{3}\right|$ and $\left|\varepsilon_{1, D_{3}}^{3}\right|$ rise monotonically with increasing of $\left|g_{D_{3} d_{3} N_{2}}^{N}\right|$. The maximum absolute values of the lepton CP asymmetries also grow when $\left|g_{D_{3} d_{3} N_{1}}^{N}\right|$ increases. At the same time $\left|\varepsilon_{1, D_{3}}^{3}\right|$ does not depend on $\left|g_{D_{3} d_{3} N_{1}}^{N}\right|$. When $\left|g_{D_{3} d_{3} N_{2}}^{N}\right| \gg\left|g_{D_{3} d_{3} N_{1}}^{N}\right|$ the decay asymmetry $\left|\varepsilon_{1, D_{3}}^{3}\right|$ tends to be considerably larger than lepton decay asymmetries. At low energies the induced lepton asymmetry in the exotic quark sector is converted into the ordinary lepton asymmetries via the decays of leptoquarks into leptons (sleptons) and ordinary quarks (squarks). In the opposite limit $\left|g_{D_{3} d_{3} N_{2}}^{N}\right| \ll\left|g_{D_{3} d_{3} N_{1}}^{N}\right|$ lepton decay asymmetries dominate over $\left|\varepsilon_{1, D_{3}}^{3}\right|$. If $\left|g_{D_{3} d_{3} N_{1}}^{N}\right| \sim\left|g_{D_{3} d_{3} N_{2}}^{N}\right|$ these CP asymmetries are comparable. From Fig. 9 one can see that the appreciable values of the decay asymmetries $\varepsilon_{1, \mu}^{3}, \varepsilon_{1, \tau}^{3}$ and $\varepsilon_{1, D_{3}}^{3} \sim 10^{-6}-10^{-4}$ can be induced if $\left|g_{D_{3} d_{3} N_{1}}^{N}\right|,\left|g_{D_{3} d_{3} N_{2}}^{N}\right| \gtrsim 0.01-0.1$. 


\subsubsection{Generation of baryon asymmetry}

Although the numerical results for the lepton decay asymmetries look very promissing it is not clear if an appropriate amount of baryon asymmetry can be generated. In order to calculate the total lepton and baryon asymmetries produced by thermal leptogenesis in the considered model the complete system of Boltzmann equations including the ones that describe the evolution of lepton number densities associated with leptoquarks should be solved. We plan to derive and analyse a complete set of Boltzmann equations within the $\mathrm{E}_{6} \mathrm{SSM}$ in the forthcoming publications.

Nevertheless there is one case which is relatively easy to analyse. It corresponds to the $\mathrm{E}_{6} \mathrm{SSM}$ with unbroken $Z_{2}^{H}$ symmetry. Indeed, in this case there is only one extra lepton doublet which interacts with the right-handed neutrinos. The Yukawa couplings of all other exotic particles to $N_{i}$ vanish in the considered limit. Then the complete set of Boltzmann equations is supplemented by only one extra equation as compared with the MSSM that describes the evolution of the lepton number density associated with extra lepton doublet $L_{4}$. As a consequence all results obtained in the SM and MSSM for the lepton and baryon asymmetries can be easily generalised in this case. In particular, one can estimate the total baryon asymmetry using an approximate formula (see [6])

$$
Y_{\Delta B} \sim 10^{-3}\left(\sum_{x=1}^{4} \varepsilon_{1, \ell_{x}}^{3} \eta_{x}\right),
$$

where $Y_{\Delta B}$ is a baryon asymmetry relative to the entropy density, i.e.

$$
Y_{\Delta B}=\left.\frac{n_{B}-n_{\bar{B}}}{s}\right|_{0}=(8.75 \pm 0.23) \times 10^{-11} .
$$

In Eq. (50) $\eta_{x}$ is an efficiency factor. A thermal population of $N_{1}$ and $\tilde{N}_{1}$ decaying completely out of equilibrium without washout effects would lead to $\eta_{x}=1$. However inverse decays and other washout processes reduce the induced asymmetries by factor $\eta_{x}$ where $\eta_{x}$ varies from 0 to 13 .

As in the SM and MSSM it is convenient to introduce a set of dimensionful parameters

$$
\begin{array}{lrl}
\widetilde{m}_{1, x}=h_{H_{3}^{u} L_{x} N_{1}}^{N} h_{H_{3}^{u} L_{x} N_{1}}^{N *} \frac{v_{2}^{2}}{M_{1}}, & \widetilde{m}_{1}=\sum_{x=1}^{4} \widetilde{m}_{1, x}, \\
m_{*}=\left.8 \pi \frac{v_{2}^{2}}{M_{1}^{2}} H\right|_{T=M_{1}}, & H\left(T=M_{1}\right)=\left.1.66 g_{*}^{1 / 2} \frac{T^{2}}{M_{P l}}\right|_{T=M_{1}},
\end{array}
$$

where $H$ is a Hubble expansion rate and $g_{*}=n_{b}+\frac{7}{8} n_{f}$ is a number of relativistic degrees of freedom in the thermal bath. Within the SM $g_{*}=106.75$ and $m_{*}=1.08 \cdot 10^{-3} \mathrm{eV}$ while

\footnotetext{
${ }^{3} \eta_{x}=0$ is the limit of $N_{1}$ interactions in perfect equilibrium so that no asymmetry is created.
} 


\begin{tabular}{|c|c|c|c|c|c|c|c|}
\hline & $\left|h_{H_{3}^{u} L_{4} N_{1}}^{N}\right|$ & $\left|h_{H_{3}^{u} L_{4} N_{2}}^{N}\right|$ & $\left|\varepsilon_{1, L_{4}}^{3}\right|_{\max }$ & $\begin{array}{c}\left|\varepsilon_{1, \tau}^{3}\right|_{\max } \\
=\left|\varepsilon_{1, \mu}^{3}\right|_{\max }\end{array}$ & $\eta_{L_{4}}$ & $\eta_{\mu}=\eta_{\tau}$ & $\left|Y_{\Delta B}\right|_{\max }$ \\
\hline$(\mathrm{A})$ & 0.1 & 1.0 & 0.0119 & $5.42 \cdot 10^{-11}$ & $3.26 \cdot 10^{-9}$ & 0.077 & $3.9 \cdot 10^{-14}$ \\
\hline$(\mathrm{B})$ & $10^{-3}$ & 1.0 & 0.0119 & $5.42 \cdot 10^{-9}$ & $3.26 \cdot 10^{-5}$ & 0.077 & $3.9 \cdot 10^{-10}$ \\
\hline$(\mathrm{C})$ & $10^{-5}$ & 1.0 & 0.00127 & $5.75 \cdot 10^{-8}$ & 0.326 & 0.077 & $4.1 \cdot 10^{-7}$ \\
\hline$(\mathrm{D})$ & 0.1 & 0.01 & $1.2 \cdot 10^{-6}$ & $5.42 \cdot 10^{-13}$ & $3.26 \cdot 10^{-9}$ & 0.077 & $3.9 \cdot 10^{-18}$ \\
\hline$(\mathrm{E})$ & $10^{-3}$ & 0.01 & $1.2 \cdot 10^{-6}$ & $5.42 \cdot 10^{-11}$ & $3.26 \cdot 10^{-5}$ & 0.077 & $3.9 \cdot 10^{-14}$ \\
\hline$(\mathrm{F})$ & $10^{-5}$ & 0.01 & $1.27 \cdot 10^{-7}$ & $5.75 \cdot 10^{-10}$ & 0.326 & 0.077 & $4.1 \cdot 10^{-11}$ \\
\hline
\end{tabular}

Table 1: Lepton decay asymmetries, efficiency factors and baryon asymmetry estimated for $M_{1}=10^{6} \mathrm{GeV}, M_{2}=10 M_{1}$ and different values of $\left|h_{H_{3}^{u} L_{4} N_{1}}^{N}\right|$ and $\left|h_{H_{3}^{u} L_{4} N_{2}}^{N}\right|$.

in the $\mathrm{E}_{6} \mathrm{SSM} g_{*}=356.25$ and $m_{*}=1.97 \cdot 10^{-3} \mathrm{eV}$. Here we concentrate on the so-called strong washout scenario when $\widetilde{m}_{1, x}>m_{*}$. In this case the efficiency factor $\eta_{x}$ for flavor $\ell_{x}$ can be estimated as (see [6])

$$
\eta_{x} \simeq \frac{m_{*}}{\widetilde{m}_{1, x}} .
$$

The results of our numerical studies are summarised in Table 1. We compute the maximal absolute values of the lepton decay asymmetries and estimate the efficiency factors as well as induced baryon asymmetry for different values of $\left|h_{H_{3}^{u} L_{4} N_{1}}^{N}\right|$ and $\left|h_{H_{3}^{u} L_{4} N_{2}}^{N}\right|$ within the see-saw models with constrained sequential dominance. As before we set $M_{1}=10^{6} \mathrm{GeV}, M_{2}=10 M_{1}$ and calculate the Yukawa couplings of the Higgs field $H_{u}$ to ordinary lepton doublets and right-handed neutrinos assuming CSD. We restrict our consideration to the part of the parameter space where $\left|\varepsilon_{1, L_{4}}^{3}\right|$ can be relatively large, i.e. $\gtrsim 10^{-6}$. This corresponds to the $\left|h_{H_{3}^{u} L_{4} N_{2}}^{N}\right| \gtrsim 0.01$ (see Fig. $5 \mathrm{~b}$ ). The results presented in Table 1 indicate that in the scenarios (B), (C) and (F) a substantial amount of baryon asymmetry may be generated. At the same time one can see that in some cases when the maximal value of $\left|\varepsilon_{1, L_{4}}^{3}\right|$ is relatively large the efficiency factor is so small that almost all lepton asymmetry is erased. Therefore the generation of relatively large lepton decay asymmetries does not guarantee the successful baryogenesis in the $\mathrm{E}_{6} \mathrm{SSM}$. This is a necessary condition but not a sufficient one. We will perform a detailed analysis of the generation of baryon and lepton asymmetries within the $\mathrm{E}_{6} \mathrm{SSM}$ in the near future.

\section{Conclusions}

In this paper we have discussed the mechanism of generation of lepton asymmetry within the Exceptional Supersymmetric Standard Model. The $\mathrm{E}_{6} \mathrm{SSM}$ is based on 
the $S U(3)_{C} \times S U(2)_{W} \times U(1)_{Y} \times U(1)_{N}$ gauge group which is a subgroup of $E_{6}$. The particle content of the Exceptional SUSY model includes three complete fundamental representations of $E_{6}$ as well as the doublet $L_{4}$ and anti-doublet $\bar{L}_{4}$ from an extra $27^{\prime}$ and $\overline{27^{\prime}}$. Thus the $\mathrm{E}_{6} \mathrm{SSM}$ involves exotic matter beyond the MSSM. In particular, it predicts the existence of three generations of exotic quarks $D_{i}$ and $\bar{D}_{i}$, and two generations of inert Higgs fields $H_{\alpha}^{d}$ and $H_{\alpha}^{u}$ that do not carry any lepton or baryon number as well as a vector-like fourth lepton doublet $L_{4}$ and $\bar{L}_{4}$ that carry a lepton number $L= \pm 1$. In the phenomenologically acceptable $E_{6}$ inspired models the extra exotic quarks can be either diquarks ( $\mathrm{E}_{6} \mathrm{SSM}$ Model $\mathrm{I}$ ) or leptoquarks ( $\mathrm{E}_{6} \mathrm{SSM}$ Model II). In order to suppress the couplings of extra inert Higgs fields to ordinary quarks and leptons that lead to the unacceptably large non-diagonal flavour transitions we imposed an approximate $Z_{2}^{H}$ symmetry.

In the $\mathrm{E}_{6} \mathrm{SSM}$ right-handed neutrinos and their superpartners do not participate in the gauge interactions and therefore can be significantly heavier than other particles from the same 27-plet. The right-handed neutrino mass scale is not fixed in the considered model but it is expected to be much lower than the Grand Unification scale. The three known doublet neutrinos $\nu_{e}, \nu_{\mu}$ and $\nu_{\tau}$ acquire small Majorana masses via the seesaw mechanism in this case. Because right-handed neutrinos are allowed to have large masses in the considered model, they may decay into the final states with lepton number $L= \pm 1$, thereby creating a lepton asymmetry in the early Universe. The dynamically induced lepton asymmetry subsequently gets converted into the observed baryon asymmetry through the electroweak phase transition. The process of the lepton asymmetry generation is controlled by the flavour dependent CP (decay) asymmetries. These decay asymmetries originate from the interference of the tree-level and one-loop amplitudes of the lightest right-handed neutrino decays. We have calculated flavour CP asymmetries within the Exceptional SUSY model and analysed their dependence on the Yukawa couplings of new exotic particles.

The new exotic particles predicted by the $\mathrm{E}_{6} \mathrm{SSM}$ contribute to the ordinary CP asymmetries induced by the decays of the lightest right-handed neutrino (or sneutrino) into the final states containing leptons and sleptons through loop diagrams. The new particles and interactions also result in new channels of the decays of the lightest right-handed neutrino and its superpartner which give rise to a set of extra decay asymmetries associated with new decay modes. When $Z_{2}^{H}$ symmetry is unbroken the only exotic particles that contribute to the generation of lepton asymmetry are the fermion and scalar components of the vector-like lepton doublet superfield $L_{4}$. In this case the analytic expressions for the flavour CP asymmetries obtained in the MSSM can be easily generalised to include $L_{4}$ since this field may be considered as a fourth generation lepton doublet. As a result 
in the $\mathrm{E}_{6} \mathrm{SSM}$ with unbroken $Z_{2}^{H}$ symmetry there are four independent $\mathrm{CP}$ asymmetries. These decay asymmetries take on non-zero values only if CP invariance is broken either in the lepton or $L_{4}$ sectors, i.e. some of the Yukawa couplings of either leptons or $L_{4}$ are complex. Our numerical analysis reveals that the absolute values of the ordinary lepton $\mathrm{CP}$ asymmetries diminish when the mass of the lightest right-handed neutrino decreases. This happens because the values of these decay asymmetries are set by the Yukawa couplings of leptons to the Higgs doublet $H_{u}$ and right-handed neutrinos which also determine the Majorana masses of the left-handed neutrino. Then the pattern of the neutrino mass-squared differences measured in the neutrino oscillation experiments imply that the lower masses of the right-handed neutrinos require the smaller values of the corresponding Yukawa couplings. As a consequence at low right-handed neutrino mass scales $\left(M_{1} \ll 10^{9} \mathrm{GeV}\right)$ the ordinary lepton decay asymmetries become extremely small.

The CP asymmetries also depend rather strongly on the Yukawa couplings of $L_{4}$. In contrast with the couplings of the ordinary lepton fields to the Higgs doublet $H_{u}$ and right-handed neutrinos the similar Yukawa couplings of $L_{4}$ are not constrained by the neutrino oscillation data because they are not related with the Majorana masses of the left-handed neutrino. Therefore these couplings can vary within a very wide range at the lightest right-handed neutrino mass scale. If the Yukawa couplings of the fourth lepton doublet superfield $L_{4}$ are large and complex they can induce a substantial CP asymmetry associated with the decay $N_{1} \rightarrow L_{4}+H_{u}\left(\left|\varepsilon_{1, L^{\prime}}\right| \sim 10^{-4}\right)$ even for relatively low $M_{1}$ (for example, $M_{1} \simeq 10^{6} \mathrm{GeV}$ ). When $M_{1} \ll 10^{13}-10^{14} \mathrm{GeV}$ this $\mathrm{CP}$ asymmetry tends to dominate over the ordinary lepton decay asymmetries. At low energies the lepton asymmetry generated in the $L_{4}$ sector gets converted into the ordinary lepton asymmetries via the decays of $L_{4}$. We have derived theoretical restrictions on the absolute values of the CP asymmetries within the see-saw models with constrained sequential dominance. The corresponding theoretical upper bounds are determined by the Yukawa couplings of the second lightest right-handed neutrino and do not depend on the Yukawa couplings of the lightest right-handed neutrino.

In the $\mathrm{E}_{6} \mathrm{SSM}$ Model I the interactions of inert Higgs superfields $H_{\alpha}^{u}$ with leptons are allowed. These interactions give rise to eight extra decay asymmetries which are not present in the $\mathrm{E}_{6} \mathrm{SSM}$ with unbroken $Z_{2}^{H}$ symmetry. We defined four total flavour CP asymmetries which correspond to three different lepton flavours $(e, \mu, \tau)$ and fourth lepton doublet $L_{4}$. It is natural to assume that inert Higgs fields $H_{\alpha}^{u}$ couple predominantly to the third generation fermions whereas the couplings of $H_{\alpha}^{u}$ to the quarks and leptons of the first two generation are negligibly small. Note that neither neutrino nor collider experiments set any limit on the Yukawa couplings of the inert-Higgs fields $H_{\alpha}^{u}$ to $\tau$-lepton and right- 
handed neutrinos so that these couplings can be of the order of 0.1 . In the considered approximation the inert-Higgs supermultiplets $H_{\alpha}^{u}$ can give a considerable contribution to the total decay asymmetry associated with $\tau$-lepton only. As before the absolute values of muon and electron CP asymmetries reduce when the mass of the lightest right-handed neutrino decreases. At low right-handed neutrino mass scales these asymmetries are negligibly small. At the same time if the Yukawa couplings of $H_{\alpha}^{u}$ to the $\tau$-lepton and right-handed neutrinos are large and complex they can induce a substantial total decay asymmetry associated with $\tau$-lepton which tends to dominate over the electron and muon ones when $M_{1} \ll 10^{13}-10^{14} \mathrm{GeV}$. We have established the theoretical upper bounds on the absolute values of the total flavour $\mathrm{CP}$ asymmetries in the framework of constrained sequential dominance. Again the maximal absolute values of the total decay asymmetries are set by the Yukawa couplings of the second lightest right-handed neutrino. Our analysis demonstrates that an appreciable $\tau$-lepton CP asymmetry $\left|\varepsilon_{1, \tau}^{\text {tot }}\right| \simeq 10^{-6}-10^{-4}$ can be generated even if $M_{1} \simeq 10^{6} \mathrm{GeV}$.

Many new decay channels of the lightest right-handed neutrino appear within the $\mathrm{E}_{6} \mathrm{SSM}$ Model II. In this model the exotic quarks and squarks carry lepton and baryon number simultaneously, i.e. they are leptoquarks. As a result the decays of the lightest right-handed neutrino into leptoquarks (squarks) and down-type squarks (quarks) are allowed. New decay modes of $N_{1}$ lead to the nine extra CP asymmetries in addition to those arising in the $\mathrm{E}_{6} \mathrm{SSM}$ Model I. Here we defined three extra total CP asymmetries which correspond to three generations of leptoquarks. The values of extra CP asymmetries are determined by the Yukawa couplings of leptoquarks which are not constrained by either neutrino or collider experiments. If these Yukawa couplings are large and complex they give rise to the appreciable $\mathrm{CP}$ asymmetries associated with the exotic quarks which are converted into the ordinary lepton asymmetries via the decays of leptoquarks into leptons (sleptons) at low energies. In the considered case the exotic quarks and squarks can also give a large contribution to the lepton CP asymmetries through loop diagrams so that a substantial values of $\varepsilon_{1, \ell_{x}}^{k}$ are induced even for the relatively low right-handed neutrino mass scales. In general the considerable values of $\mathrm{CP}$ asymmetries $\sim 10^{-6}-10^{-4}$ can be induced independently of the right-handed neutrino mass scale if new Yukawa couplings of the right-handed neutrino vary from 0.01 to 0.1 .

Thus the results presented in this paper show that substantial asymmetries can be generated even for very low right-handed neutrino masses of order $10^{6} \mathrm{GeV}$. We have also briefly considered the efficiency factors relevant for the case of the $\mathrm{E}_{6} \mathrm{SSM}$ with unbroken $Z_{2}^{H}$ symmetry, and shown that acceptably large baryon asymmetries can result. These results suggest that in the $\mathrm{E}_{6} \mathrm{SSM}$ successful thermal leptogenesis can be achieved without encountering problems with gravitinos. 


\section{Acknowledgements}

We would like to thank S. Antusch, C. D. Froggatt, G. G. Ross, S. Sarkar, J. MarchRussell, L. B. Okun, P. Soler and D. Sutherland for fruitful discussions. RN acknowledge support from the SHEFC grant HR03020 SUPA 36878. SFK acknowledges partial support from the following grants: PPARC Rolling Grant PPA/G/S/2003/00096; EU Network MRTN-CT-2004-503369; NATO grant PST.CLG.980066; EU ILIAS RII3-CT2004-506222. 


\section{Appendix: CP asymmetries in the effective field theory approach}

In the presence of a large mass gap between the lightest and second lightest right-handed neutrinos one can use the effective field theory approach for the calculation of CP asymmetries. Assuming that $M_{2}, M_{3} \gg M_{1}$ one can integrate out two heavy right-handed neutrinos so that at the energies below $M_{2}$ but above $M_{1}$ the interactions between superfields are described by the effective superpotential:

$$
\begin{aligned}
W_{\mathrm{eff}} & \simeq h_{k x 1}^{N}\left(H_{k}^{u} L_{x}\right) N_{1}^{c}+g_{k i 1}^{N}\left(D_{k} d_{i}^{c}\right) N_{1}^{c}+\frac{1}{2} \Xi_{k x i y}^{L L}\left(H_{k}^{u} L_{x}\right)\left(H_{i}^{u} L_{y}\right) \\
& +\Xi_{k x m p}^{L D}\left(H_{k}^{u} L_{x}\right)\left(D_{m} d_{p}^{c}\right)+\frac{1}{2} \Xi_{k i m p}^{D D}\left(D_{k} d_{i}^{c}\right)\left(D_{m} d_{p}^{c}\right)+\ldots,
\end{aligned}
$$

where $i, k, m, p$ are family indexes that run from 1 to 3 while $x$ and $y$ vary from 1 to 4 and

$$
\begin{gathered}
\Xi_{k x i y}^{L L}=\Xi_{i y k x}^{L L}=-\sum_{j=2,3} \frac{h_{k x j}^{N} h_{i y j}^{N}}{M_{j}}, \quad \Xi_{k x m p}^{L D}=-\sum_{j=2,3} \frac{h_{k x j}^{N} g_{m p j}^{N}}{M_{j}}, \\
\Xi_{k i m p}^{D D}=\Xi_{m p k i}^{D D}=-\sum_{j=2,3} \frac{g_{k i j}^{N} g_{m p j}^{N}}{M_{j}} .
\end{gathered}
$$

The last three terms in the superpotential (A.1) are the lowest dimensional effective operators induced by the heavy right-handed neutrinos. In the effective field theory approach these operators give rise to the non-zero values of the $\mathrm{CP}$ asymmetries which originate from the interference of the tree-level and one-loop amplitudes of the lightest right-handed neutrino decays. The associated one-loop diagrams involve two vertices one of which represents non-renormalisable lepton number violating interactions induced by the heavy right-handed neutrinos. Calculating one-loop diagrams, we find

$$
\begin{aligned}
\varepsilon_{1, \ell_{x}}^{k} & \simeq \frac{M_{1}}{8 \pi A_{1}} \operatorname{Im}\left[2 \sum_{i, y} h_{k x 1}^{N *} \Xi_{k x i y}^{L L} h_{i y 1}^{N *}+\sum_{m, p} h_{k x 1}^{N *} \Xi_{i x k y}^{L L} h_{i y 1}^{N *}\right. \\
& \left.+3 \sum_{m, p} h_{k x 1}^{N *} \Xi_{k x m p}^{L D} g_{m p 1}^{N *}\right], \\
\varepsilon_{1, D_{k}}^{i} & \simeq \frac{M_{1}}{8 \pi A_{0}} \operatorname{Im}\left[3 \sum_{m, p} g_{k i 1}^{N *} \Xi_{k i m p}^{D D} g_{m p 1}^{N *}+\sum_{m, p} g_{k i 1}^{N *} \Xi_{m i k p}^{D D} g_{m p 1}^{N *}\right. \\
& \left.+2 \sum_{m, p} g_{k i 1}^{N *} \Xi_{m y k i}^{L D} h_{m y 1}^{N *}\right]
\end{aligned}
$$

In the case of the $\mathrm{E}_{6} \mathrm{SSM}$ version I, $g_{m p j}^{N}$, $\Xi_{k i m p}^{D D}$ and $\Xi_{k x i m}^{L D}$ vanish and the expressions for the decay asymmetries (A.3) are simplified drastically. In particular, $\varepsilon_{1, D_{k}}^{i}$ tend to zero because in the considered case the exotic quarks are diquarks and therefore the baryon number conservation forbids the decay of the lightest right-handed neutrino into exotic quarks (or squarks). The Eqs. (A.3) can be simplified even further if one sets $h_{1 x j}^{N}=h_{2 x j}^{N}=0$. Then the results for the CP asymmetries (18) derived in the exact $Z_{2}^{H}$ 
symmetry limit are reproduced. Finally, the Eqs. (A.3) can be obtained directly from Eqs. (13) and (24) by setting $\left(M_{1} / M_{j}\right) \rightarrow 0$. One can easily check that in the considered limit the analytic expressions for the decay asymmetries (13) and (24) coincide with the results obtained in the effective field theory approximation.

\section{References}

[1] E. Komatsu et al. [WMAP Collaboration], arXiv:0803.0547 [astro-ph].

[2] A. Yu. Ignatiev, N. V. Krasnikov, V. A. Kuzmin, and A. N. Tavkhelidze, Phys. Lett. B 76 (1978) 436; M. Yoshimura, Phys. Rev. Lett. 41 (1978) 281; D. Toussaint, S. B. Treiman, F. Wilczek, and A. Zee, Phys. Rev. D 19 (1979) 1036; Steven Weinberg, Phys. Rev. Lett. 42 (1979) 850; M. Yoshimura, Phys. Lett. B 88 (1979) 294; S. M. Barr, G. Segre and H. A. Weldon, Phys. Rev. D 20 (1979) 2494; D. V. Nanopoulos, S. Weinberg, Phys. Rev. D 20 (1979) 2484; A. Yildiz, P. Cox, Phys. Rev. D 21 (1980) 906 .

[3] A. Riotto and M. Trodden, Ann. Rev. Nucl. Part. Sci. (1999) 49 35; J. M. Cline. hep-ph/0609145.

[4] S. J. Huber and M. G. Schmidt, Nucl. Phys. B 606 (2001) 183.

[5] M. Fukugita and T. Yanagida, Phys. Lett. B 174 (1986) 45.

[6] for a recent review, see S. Davidson, E. Nardi and Y. Nir, arXiv:0802.2962.

[7] I. Affleck and M. Dine. Nucl. Phys. B 249 (1985) 361; M. Dine, L. Randall and S. D. Thomas, Nucl. Phys. B 458 (1996) 291.

[8] P. Minkowski, Phys. Lett. B 67 (1977) 421; M. Gell-Mann, P. Ramond and R. Slansky, Proceedings of the Supergravity Stony Brook Workshop, New York 1979, eds. P. Van Nieuwenhuizen and D. Freedman; T. Yanagida, Proceedings of the Workshop on Unified Theory and Baryon Number in the Universe, Tsukuba, Japan 1979, eds. A. Sawada and A. Sugamoto; R. N. Mohapatra, G. Senjanovic, Phys. Rev. Lett. 44 (1980) 912 .

[9] A. D. Sakharov, Pisma Zh. Eksp. Teor. Fiz. 5 (1967) 32 [JETP Lett. 5 (1967) 24].

[10] V. A. Kuzmin, V. A. Rubakov and M. E. Shaposhnikov, Phys. Lett. B 155 (1985) 36; V. A. Rubakov and M. E. Shaposhnikov, Usp. Fiz. Nauk, 166 (1996) 493. 
[11] M. A. Luty, Phys. Rev. D 45 (1992) 455; M. Flanz, E. A. Paschos and U. Sarkar, Phys. Lett. B 345 (1995) 248 [Erratum-ibid. B 382 (1996) 447]; M. Plumacher, Z. Phys. C 74 (1997) 549; W. Buchmuller and M. Plumacher, Phys. Lett. B 431 (1998) 354.

[12] B. A. Campbell, S. Davidson and K. A. Olive, Nucl. Phys. B 399 (1993) 111; L. Covi, E. Roulet and F. Vissani, Phys. Lett. B 384 (1996) 169; M. Plumacher, Nucl. Phys. B 530 (1998) 207.

[13] G. F. Giudice, A. Notari, M. Raidal, A. Riotto, and A. Strumia, Nucl. Phys. B 685 (2004) 89; W. Buchmuller, P. Di Bari, and M. Plumacher, Ann. Phys. 315 (2005) 305.

[14] R. Barbieri, P. Creminelli, A. Strumia, and N. Tetradis, Nucl. Phys. B 575 (2000) 61; T. Endoh, T. Morozumi, and Z. h. Xiong, Prog. Theor. Phys. 111 (2004) 123; O. Vives, Phys. Rev. D 73 (2006) 073006; A. Abada, S. Davidson, F. X. JosseMichaux, M. Losada and A. Riotto, JCAP 0604 (2006) 004; E. Nardi, Y. Nir, E. Roulet and J. Racker, JHEP 0601 (2006) 164; A. Abada, S. Davidson, A. Ibarra, F. X. Josse-Michaux, M. Losada and A. Riotto, JHEP 0609 (2006) 010; A. De Simone and A. Riotto, JCAP 0702 (2007) 005; S. Blanchet, P. Di Bari and G. G. Raffelt, JCAP 0703 (2007) 012.

[15] S. Antusch, S. F. King and A. Riotto, JCAP 0611 (2006) 011.

[16] E. Ma and U. Sarkar, Phys. Rev. Lett. 80 (1998) 5716; T. Hambye, E. Ma, and U. Sarkar, Nucl. Phys. B 602 (2001) 23; E. J. Chun and S. K. Kang, Phys. Rev. D 63 (2001) 097902; A. S. Joshipura, E. A. Paschos, and W. Rodejohann, Nucl. Phys., B611:227-238, 2001; B. Brahmachari, E. Ma, and U. Sarkar, Phys. Lett. B 520 (2001) 152; T. Hambye and G. Senjanovic, Phys. Lett. B 582 (2004) 73; W. l. Guo, Phys. Rev. D 70 (2004) 053009; S. Antusch and S. F. King, Phys. Lett. B 597 (2004) 199; S. Antusch and S. F. King, JHEP 0601 (2006) 117; T. Hambye, M. Raidal, and A. Strumia, Phys. Lett. B 632 (2006) 667; E. J. Chun and S. Scopel, Phys. Rev. D 75 (2007) 023508; S. Antusch, Phys. Rev. D 76 (2007) 023512; W. Chao, S. Luo and Z. z. Xing, Phys. Lett. B 659 (2008) 281; T. Hallgren, T. Konstandin and T. Ohlsson, JCAP 0801 (2008) 014.

[17] G. D’Ambrosio, T. Hambye, A. Hektor, M. Raidal, and A. Rossi, Phys. Lett. B 604 (2004) 199; E. J. Chun and S. Scopel, Phys. Lett. B 636 (2006) 278.

[18] K. Dick, M. Lindner, M. Ratz, and D. Wright, Phys. Rev. Lett. 84 (2000) 4039; H. Murayama and A. Pierce, Phys. Rev. Lett. 89 (2002) 271601; M. Boz and 
N. K. Pak, Eur. Phys. J. C 37 (2004) 507; D. G. Cerdeno, A. Dedes, and T. E. J. Underwood, JHEP 0609 (2006) 067; B. Thomas and M. Toharia, Phys. Rev. D 73 (2006) 063512; B. Thomas and M. Toharia, Phys. Rev. D 75 (2007) 013013.

[19] S. Davidson and A. Ibarra, Phys. Lett. B 535 (2002) 25; K. Hamaguchi, H. Murayama and T. Yanagida, Phys. Rev. D 65 (2002) 043512.

[20] M. Yu. Khlopov and A. D. Linde, Phys. Lett. B 138 (1984) 265; J. R. Ellis, J E. Kim, and D. V. Nanopoulos, Phys. Lett. B 145 (1984) 181.

[21] M. Y. Khlopov, Yu. L. Levitan, E. V. Sedelnikov and I. M. Sobol, Phys. Atom. Nucl. 57 (1994) 1393 [Yad. Fiz. 57 (1994) 1466]; M. Kawasaki, K. Kohri and T. Moroi, Phys. Rev. D 71 (2005) 083502; K. Kohri, T. Moroi and A. Yotsuyanagi, Phys. Rev. D 73 (2006) 123511.

[22] Y. Grossman, T. Kashti, Y. Nir and E. Roulet, Phys. Rev. Lett. 91 (2003) 251801; G. D’Ambrosio, G. F. Giudice and M. Raidal, Phys. Lett. B 575 (2003) 75; Y. Grossman, T. Kashti, Y. Nir and E. Roulet, JHEP 0411 (2004) 080; E. J. Chun, Phys. Rev. D 69 (2004) 117303; L. Boubekeur, T. Hambye and G. Senjanovic, Phys. Rev. Lett. 93 (2004) 111601; M. C. Chen and K. T. Mahanthappa, Phys. Rev. D 70 (2004) 113013; T. Kashti, Phys. Rev. D 71 (2005) 013008; Y. Grossman, R. Kitano and H. Murayama, JHEP 0506 (2005) 058; A. D. Medina and C. E. M. Wagner, JHEP 0612 (2006) 037; J. Garayoa, M. C. Gonzalez-Garcia and N. Rius, JHEP 0702 (2007) 021; E. J. Chun and L. Velasco-Sevilla, JHEP 0708 (2007) 075. S. Dar, S. J. Huber, V. N. Senoguz and Q. Shafi, Phys. Rev. D 69 (2004) 077701;

[23] A. Pilaftsis and T. E. J. Underwood, Nucl. Phys. B 692 (2004) 303; T. Hambye, J. March-Russell and S. M. West, JHEP 0407 (2004) 070; C. H. Albright and S. M. Barr, Phys. Rev. D 70 (2004) 033013; S. Dar, S. J. Huber, V. N. Senoguz and Q. Shafi, Phys. Rev. D 69 (2004) 077701; A. Pilaftsis and T. E. J. Underwood, Phys. Rev. D 72 (2005) 113001; A. Pilaftsis, Phys. Rev. Lett. 95 (2005) 081602; A. Anisimov, A. Broncano and M. Plumacher, Nucl. Phys. B 737 (2006) 176; S. M. West, Mod. Phys. Lett. A 21 (2006) 1629; Z. z. Xing and S. Zhou, Phys. Lett. B 653 (2007) 278; A. De Simone and A. Riotto, JCAP 0708 (2007) 013; V. Cirigliano, A. De Simone, G. Isidori, I. Masina and A. Riotto, JCAP 0801 (2008) 004.

[24] T. Hambye, Nucl. Phys. B 633 (2002) 171; A. Abada and M. Losada, Nucl. Phys. B 673 (2003) 319; A. Abada, H. Aissaoui and M. Losada, Nucl. Phys. B 728 (2005) 55; A. Abada, G. Bhattacharyya and M. Losada, Phys. Rev. D 73 (2006) 033006; D. Atwood, S. Bar-Shalom and A. Soni, Phys. Lett. B 635 (2006) 112; M. Frigerio, 
T. Hambye and E. Ma, JCAP 0609 (2006) 009; E. Ma, N. Sahu and U. Sarkar, J. Phys. G 34 (2007) 741; M. Hirsch, J. W. F. Valle, M. Malinsky, J. C. Romao and U. Sarkar, Phys. Rev. D 75 (2007) 011701; N. Sahu and U. Sarkar, Phys. Rev. D 76 (2007) 045014.

[25] G. Lazarides and Q. Shafi, Phys. Lett. B 258 (1991) 305; T. Asaka, K. Hamaguchi, M. Kawasaki and T. Yanagida. Phys. Lett. B 464 (1999) 12.

[26] G. F. Giudice, M. Peloso, A. Riotto and I. Tkachev, JHEP 9908 (1999) 014.

[27] M. Bolz, W. Buchmuller and M. Plumacher, Phys. Lett. B 443 (1998) 209; J. L. Feng, S. Su and F. Takayama, Phys. Rev. D 70 (2004) 075019; T. Kanzaki, M. Kawasaki, K. Kohri and T. Moroi, Phys. Rev. D 75 (2007) 025011.

[28] M. Ibe, R. Kitano, H. Murayama and T. Yanagida, Phys. Rev. D 70 (2004) 075012.

[29] S. F. King, S. Moretti and R. Nevzorov, Phys. Rev. D 73 (2006) 035009.

[30] S. F. King, S. Moretti and R. Nevzorov, Phys. Lett. B 634 (2006) 278.

[31] T. Hambye, E. Ma, M. Raidal and U. Sarkar, Phys. Lett. B 512 (2001) 373.

[32] S. F. King, Phys. Lett. B 439 (1998) 350; S. F. King, Nucl. Phys. B 562 (1999) 57; S. F. King, Nucl. Phys. B 576 (2000) 85; S. F. King, JHEP 0209 (2002) 011.

[33] S. F. King, JHEP 0209 (2002) 011.

[34] S. F. King, Phys. Rev. D 67 (2003) 113010.

[35] Y. Kawamura, Prog. Theor. Phys. 105 (2001) 999; G. Altarelli and F. Feruglio, Phys. Lett. B 511 (2001) 257; L. J. Hall and Y. Nomura, Phys. Rev. D 64 (2001) 055003; A. Hebecker and J. March-Russell, Nucl. Phys. B 613 (2001) 3; T. Asaka, W. Buchmuller and L. Covi, Phys. Lett. B 523 (2001) 199; L. J. Hall, Y. Nomura, T. Okui and D. R. Smith, Phys. Rev. D 65 (2002) 035008.

[36] S. Kraml et al. (eds.), Workshop on CP studies and non-standard Higgs physics, CERN-2006-009, hep-ph/0608079; S.F. King, S. Moretti, R. Nevzorov, hep-ph/0601269; S.F. King, S. Moretti, R. Nevzorov, hep-ph/0610002; R. Howl and S. F. King, JHEP 0801 (2008) 030.

[37] S. F. King, S. Moretti and R. Nevzorov, Phys. Lett. B 650 (2007) 57.

[38] R. Howl and S. F. King, arXiv:0802.1909 [hep-ph]. 
[39] J. Rich, M. Spiro, J. Lloyd-Owen, Phys. Rept. 151 (1987) 239; P.F. Smith, Contemp. Phys. 29 (1988) 159; T.K. Hemmick et al., Phys. Rev. D 41 (1990) 2074.

[40] K. S. Babu, C. N. Leung and J. T. Pantaleone, Phys. Lett. B 319 (1993) 191 arXiv:hep-ph/9309223]; J. R. Ellis, G. K. Leontaris, S. Lola and D. V. Nanopoulos, Eur. Phys. J. C 9 (1999) 389 [arXiv:hep-ph/9808251]; A. Dighe, S. Goswami and P. Roy, Phys. Rev. D 76 (2007) 096005.

[41] B. Pontecorvo, Zh. Eksp. Teor. Fiz. 33 (1957) 549; ibid. 34 (1958) 247; ibid. 53 (1967) 1717; Z. Maki, M. Nakagawa and S. Sakata, Prog. Theor. Phys. 28 (1962) 870.

[42] M. Apollonio et al. [CHOOZ Collaboration], Phys. Lett. B 466 (1999) 415.

[43] S. F. King and N. N. Singh, Nucl. Phys. B 591 (2000) 3.

[44] P. F. Harrison, D. H. Perkins and W. G. Scott, Phys. Lett. B 458 (1999) 79; P. F. Harrison, D. H. Perkins and W. G. Scott, Phys. Lett. B 530 (2002) 167; P. F. Harrison and W. G. Scott, Phys. Lett. B 535 (2002) 163; P. F. Harrison and W. G. Scott, Phys. Lett. B 557 (2003) 76; C. I. Low and R. R. Volkas, Phys. Rev. D 68 (2003) 033007. A similar but physically different form was proposed earlier by: L. Wolfenstein, Phys. Rev. D 18 (1978) 958.

[45] S. F. King, JHEP 0508 (2005) 105.

[46] G. L. Fogli, E. Lisi, A. Marrone and A. Palazzo, Prog. Part. Nucl. Phys. 57 (2006) 742. 


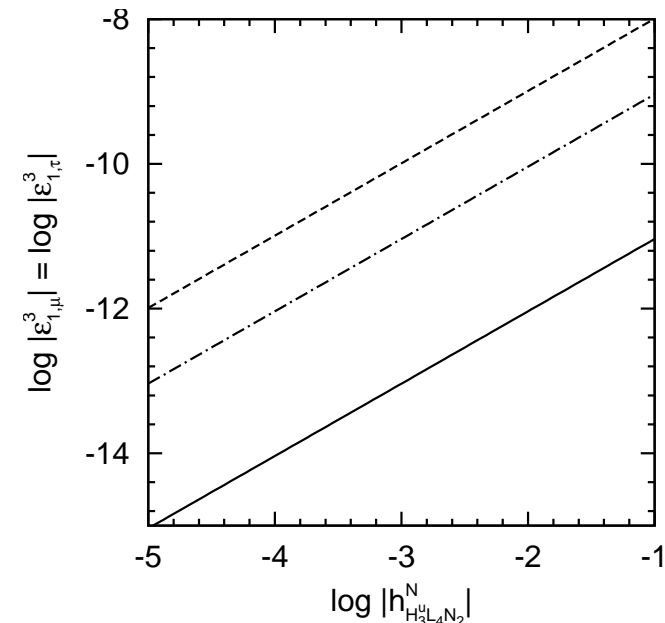

(a)

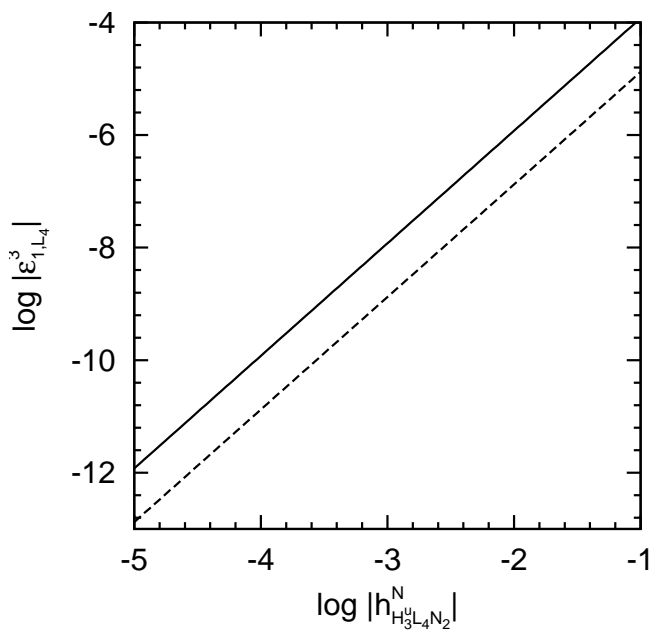

(c)

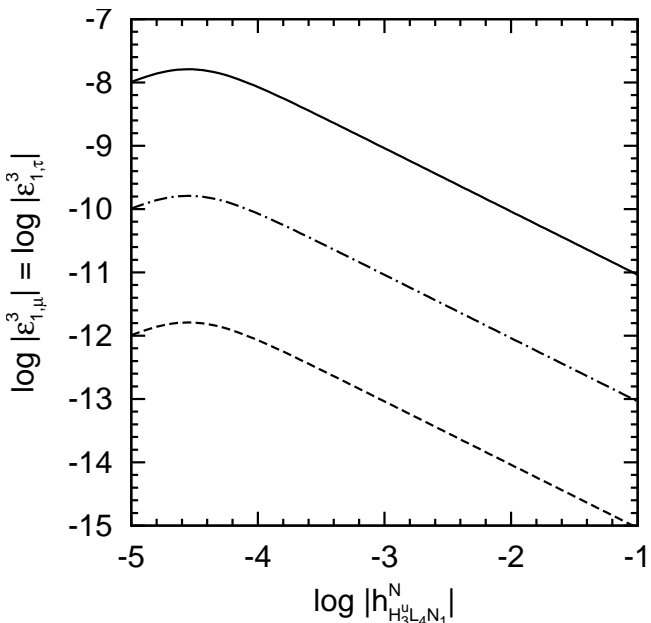

(b)

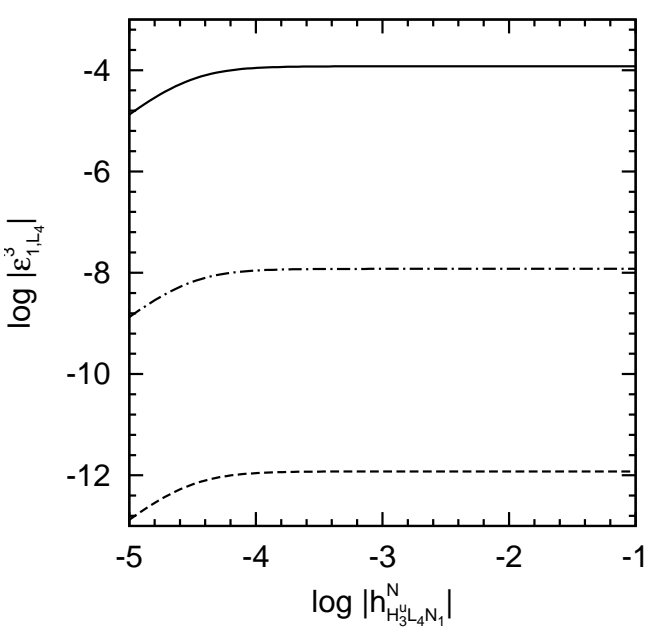

(d)

Figure 4: Maximal absolute values of $(a)-(b)\left|\varepsilon_{1, \mu}^{3}\right|=\left|\varepsilon_{1, \tau}^{3}\right|$ and (c)-(d) $\left|\varepsilon_{1, L_{4}}^{3}\right|$ in the $\mathrm{E}_{6} \mathrm{SSM}$ with unbroken $Z_{H}^{2}$ symmetry versus $\left|h_{H_{3}^{u} L_{4} N_{1}}^{N}\right|$ and $\left|h_{H_{3}^{u} L_{4} N_{2}}^{N}\right|$ for $M_{1}=10^{6} \mathrm{GeV}, M_{2}=10 \cdot M_{1}$. The solid, dash-dotted and dashed lines in figures (a) and (c) represent the maximal absolute values of the decay asymmetries for $\left|h_{H_{3}^{u} L_{4} N_{1}}^{N}\right|=0.1,10^{-3}$ and $10^{-5}$ while solid, dash-dotted and dashed lines in figures (b) and (d) correspond to $\left|h_{H_{3}^{u} L_{4} N_{2}}^{N}\right|=0.1,10^{-3}$ and $10^{-5}$ respectively. 


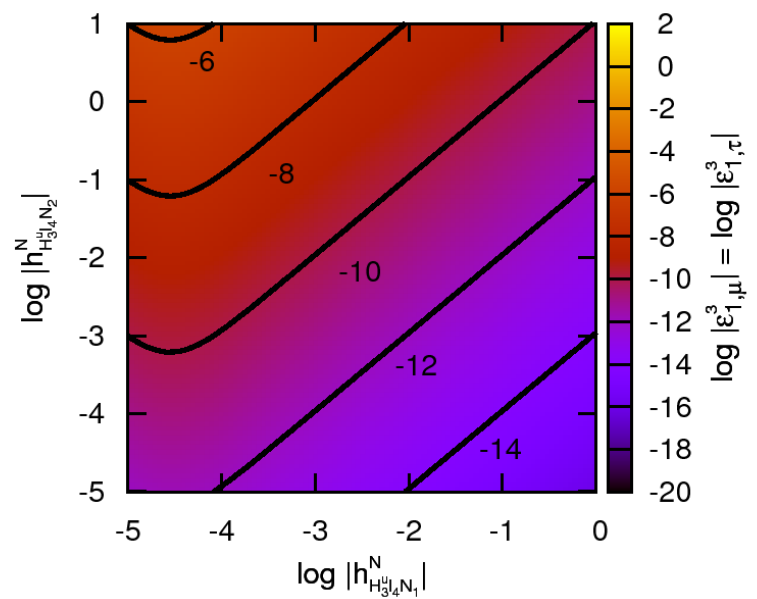

(a)

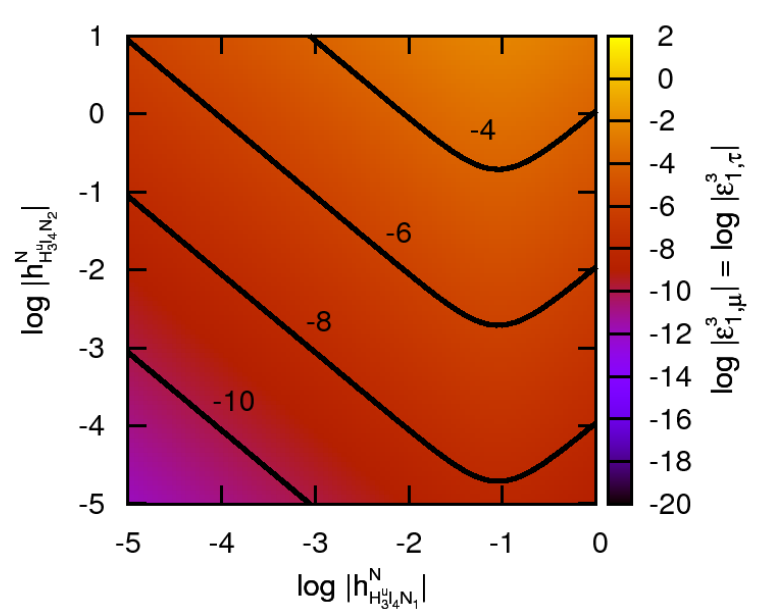

(c)

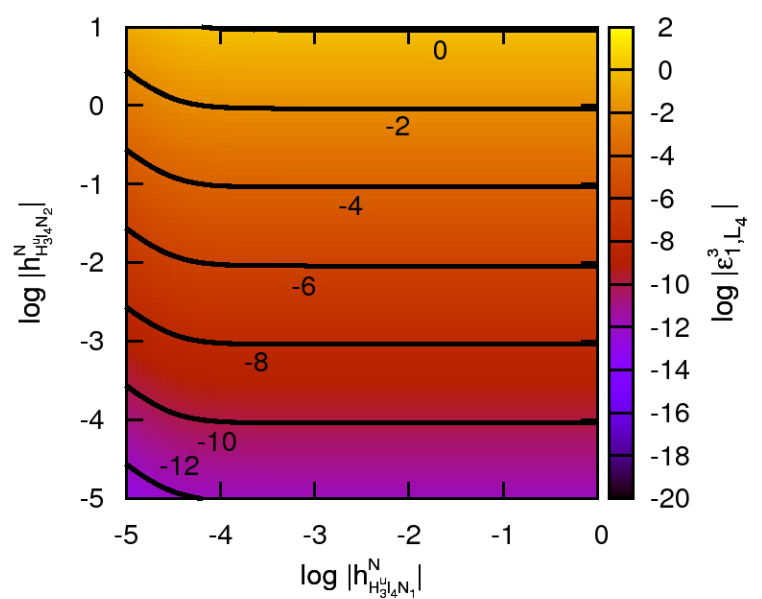

(b)

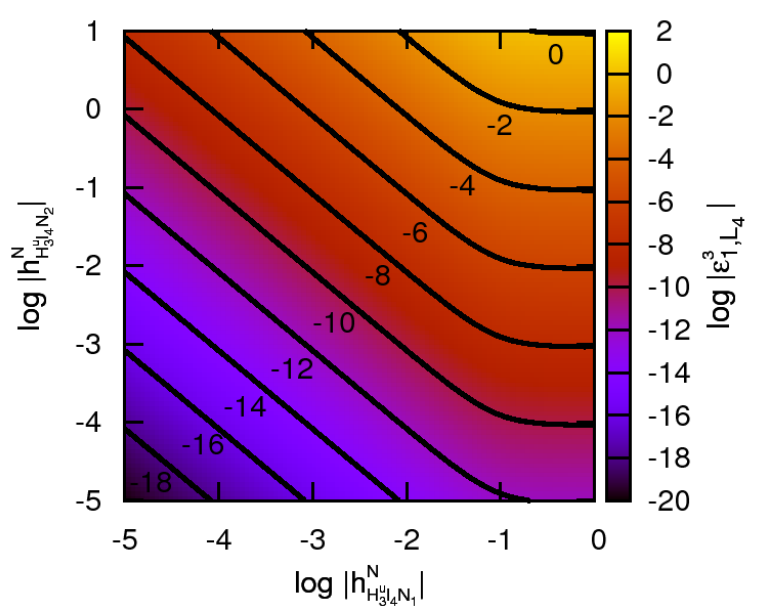

(d)

Figure 5: Logarithm (base 10) of the maximal values of $\left|\varepsilon_{1, \mu}^{3}\right|=\left|\varepsilon_{1, \tau}^{3}\right|(a, c)$ and $\left|\varepsilon_{1, L_{4}}^{3}\right|(b, d)$ in the $\mathrm{E}_{6} \mathrm{SSM}$ with unbroken $Z_{H}^{2}$ symmetry versus $\log \left|h_{H_{3}^{u} L_{4} N_{1}}^{N}\right|$ and $\log \left|h_{H_{3}^{u} L_{4} N_{2}}^{N}\right|$ for $M_{1}=10^{6} \mathrm{GeV}(a, b)$, $M_{1}=10^{13} \mathrm{GeV}(c, d)$, and $M_{2}=10 M_{1}$. The solid contour lines show steps of 2 in the logarithm (base 10) of the asymmetries. 


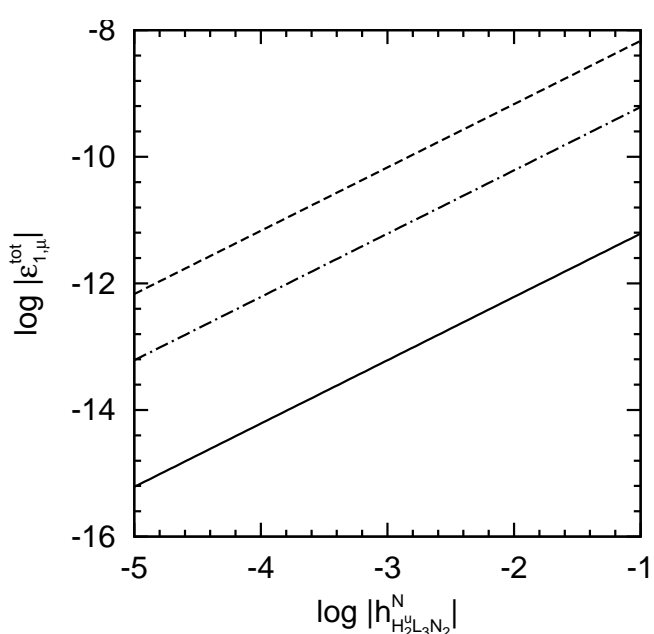

(a)

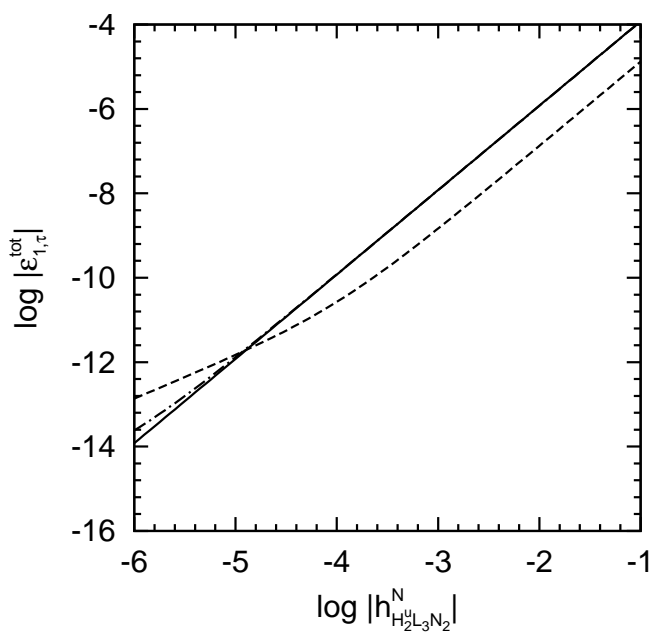

(c)

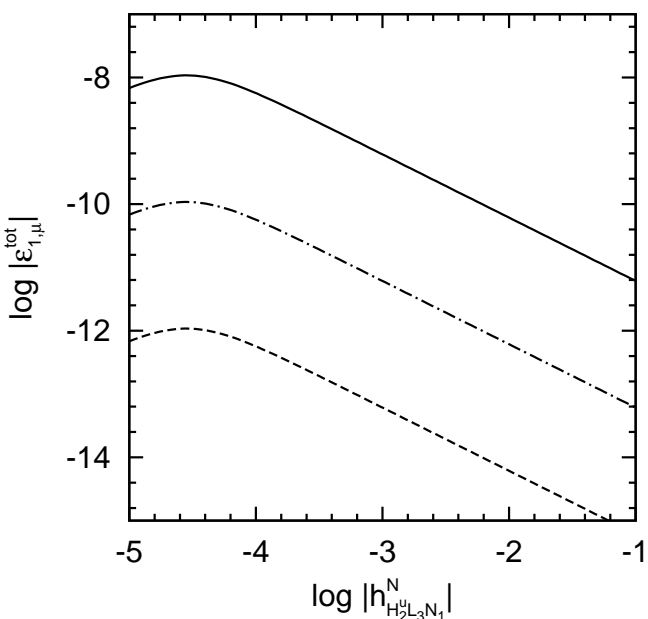

(b)

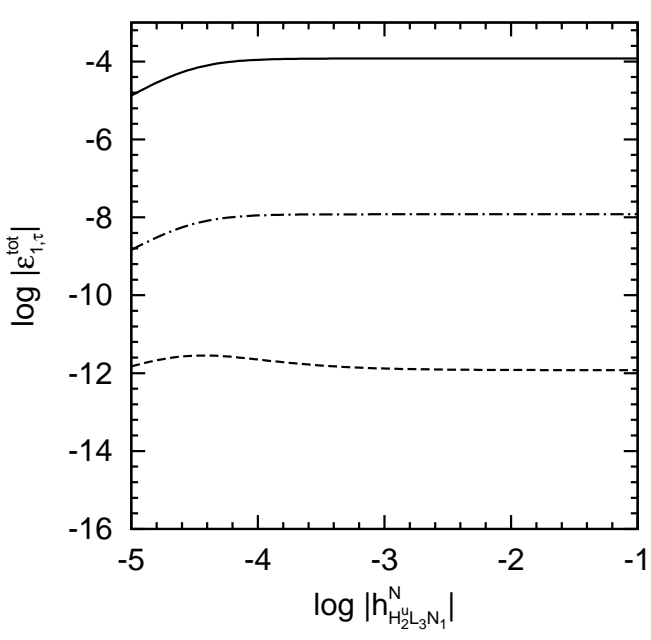

(d)

Figure 6: Maximal absolute values of $(a)-(b)\left|\varepsilon_{1, \mu}^{t o t}\right|$ and $(c)-(d)\left|\varepsilon_{1, \tau}^{t o t}\right|$ in the $\mathrm{E}_{6} \mathrm{SSM}$ Model I versus $\left|h_{H_{2}^{u} L_{3} N_{2}}^{N}\right|$ and $\left|h_{H_{2}^{u} L_{3} N_{1}}^{N}\right|$ for $M_{1}=10^{6} \mathrm{GeV}$ and $M_{2}=10 \cdot M_{1}$. All couplings $\left|h_{H_{k}^{u} L_{4} N_{j}}^{N}\right|$ are set to zero. The solid, dash-dotted and dashed lines in figures (a) and (c) represent the maximal absolute values of the decay asymmetries for $\left|h_{H_{2}^{u} L_{3} N_{1}}^{N}\right|=0.1,10^{-3}$ and $10^{-5}$ while solid, dash-dotted and dashed lines in figures (b) and (d) correspond to $\left|h_{H_{2}^{u} L_{3} N_{2}}^{N}\right|=0.1,10^{-3}$ and $10^{-5}$ respectively. 


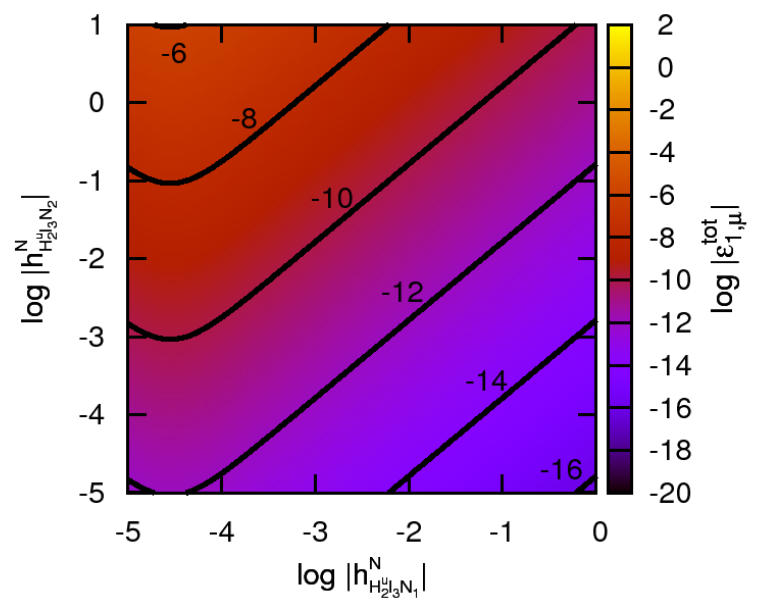

(a)

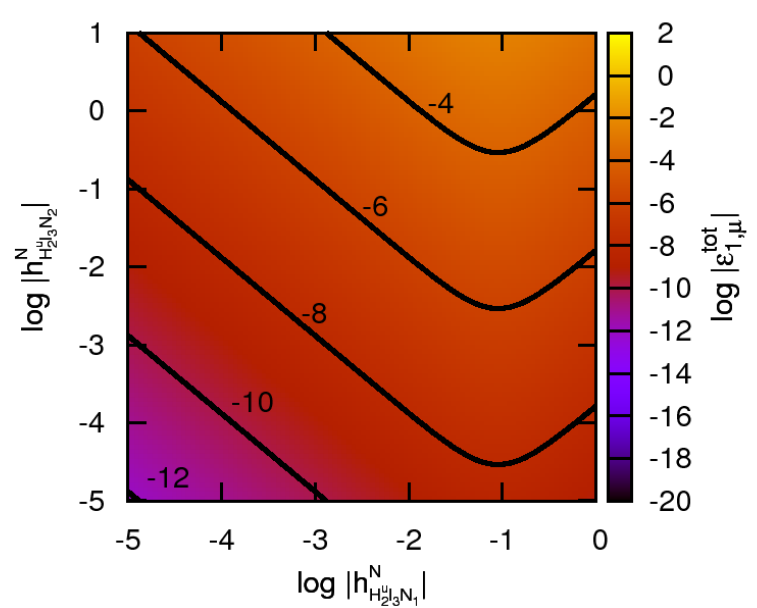

(c)

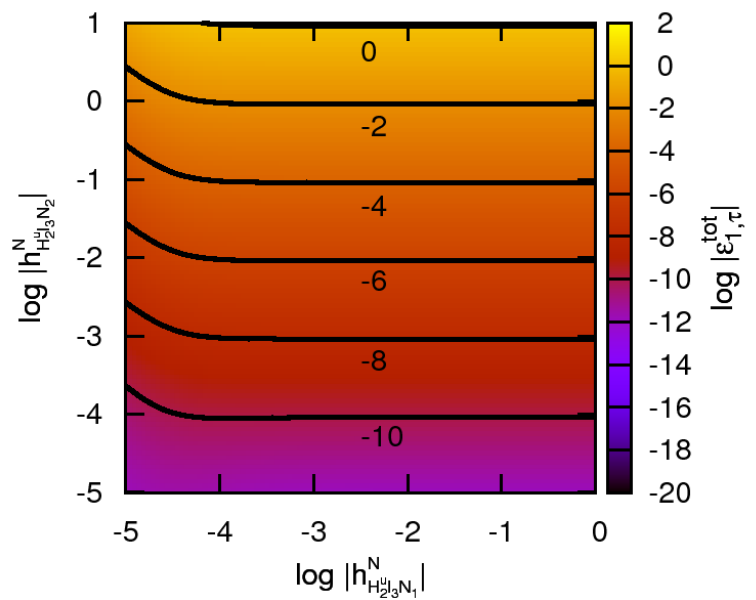

(b)

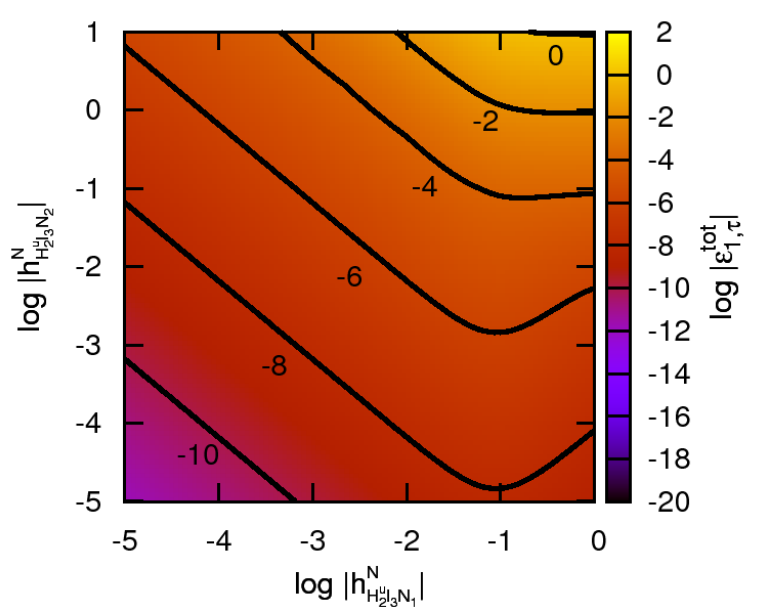

(d)

Figure 7: Logarithm (base 10) of the maximal values of $\left|\varepsilon_{1, \mu}^{t o t}\right|(a, c)$ and $\left|\varepsilon_{1, \tau}^{t o t}\right|(b, d)$ in the $\mathrm{E}_{6} \mathrm{SSM}$ Model I versus $\log \left|h_{H_{2}^{u} L_{3} N_{2}}^{N}\right|$ and $\log \left|h_{H_{2}^{u} L_{3} N_{1}}^{N}\right|$ for $M_{1}=10^{6} \mathrm{GeV}(a, b), M_{1}=10^{13} \mathrm{GeV}(c, d)$, and $M_{2}=10 M_{1}$. All couplings $\left|h_{H_{k}^{u} L_{4} N_{j}}^{N}\right|$ are set to zero. The solid contour lines show steps of 2 in the logarithm (base 10) of the asymmetries. 


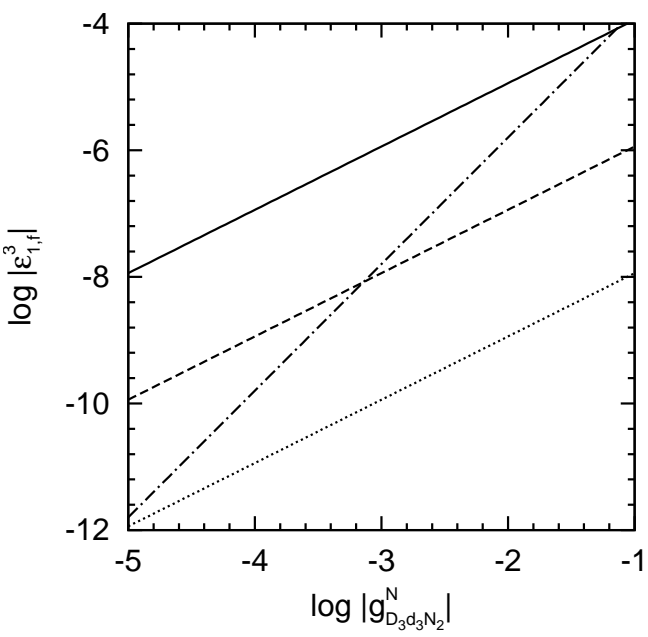

(a)

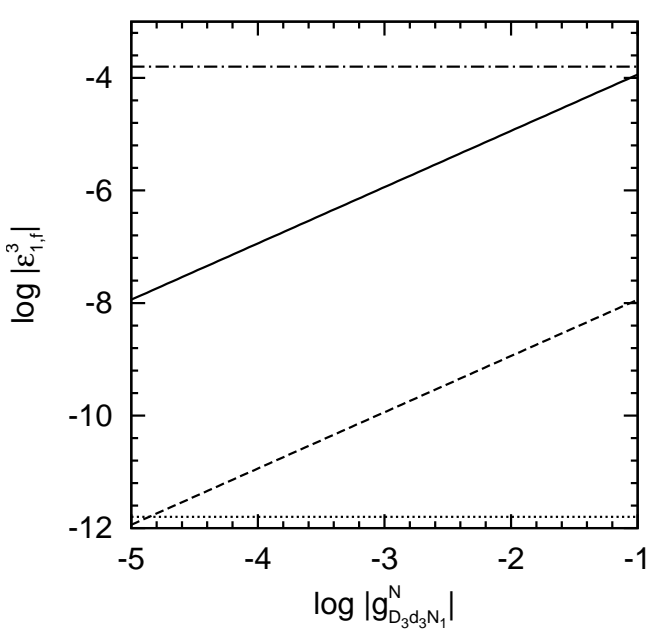

(b)

Figure 8: Maximal absolute values of the $\mathrm{CP}$ asymmetries in the $\mathrm{E}_{6} \mathrm{SSM}$ Model II as a function of (a) $\left|g_{D_{3} d_{3} N_{2}}^{N}\right|$ and $(b)\left|g_{D_{3} d_{3} N_{1}}^{N}\right|$ for $M_{1}=10^{6} \mathrm{GeV}$ and $M_{2}=10 \cdot M_{1}$. All couplings $\left|h_{H_{k}^{u} L_{4} N_{j}}^{N}\right|$ and $\left|h_{H_{\alpha}^{u} L_{x} N_{j}}^{N}\right|$ $(\alpha=1,2)$ are set to zero. The solid, dashed and dotted lines in figure (a) represent $\left|\varepsilon_{1, \mu}^{3}\right|=\left|\varepsilon_{1, \tau}^{3}\right|$ computed for $\left|g_{D_{3} d_{3} N_{1}}^{N}\right|=0.1,10^{-3}$ and $10^{-5}$ while the dash-dotted line corresponds to $\left|\varepsilon_{1, D_{3}}^{3}\right|$. The solid and dashed lines in figure (b) show the dependence of $\left|\varepsilon_{1, \mu}^{3}\right|=\left|\varepsilon_{1, \tau}^{3}\right|$ on $\left|g_{D_{3} d_{3} N_{1}}^{N}\right|$ for $\left|g_{D_{3} d_{3} N_{2}}^{N}\right|=0.1$ and $10^{-5}$ while the dash-dotted and dotted lines correspond to $\left|\varepsilon_{1, D_{3}}^{3}\right|$ calculated for $\left|g_{D_{3} d_{3} N_{2}}^{N}\right|=0.1$ and $10^{-5}$ respectively.

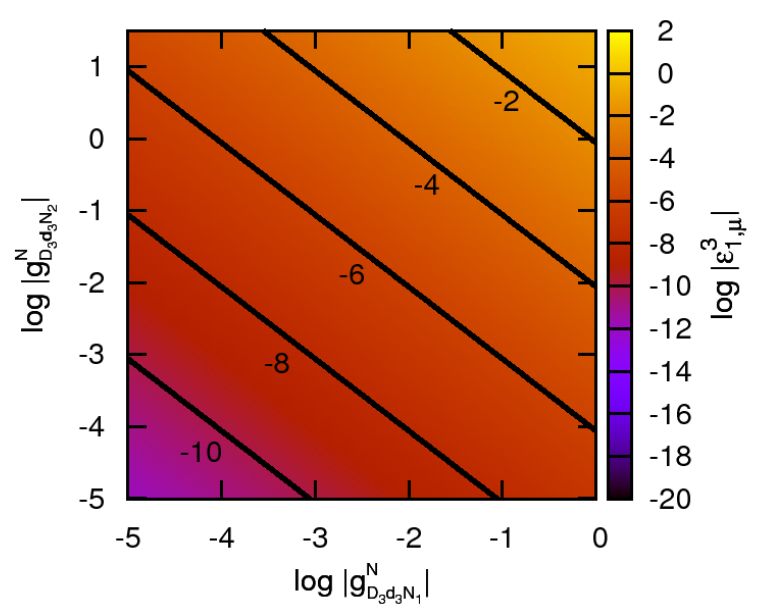

(a)

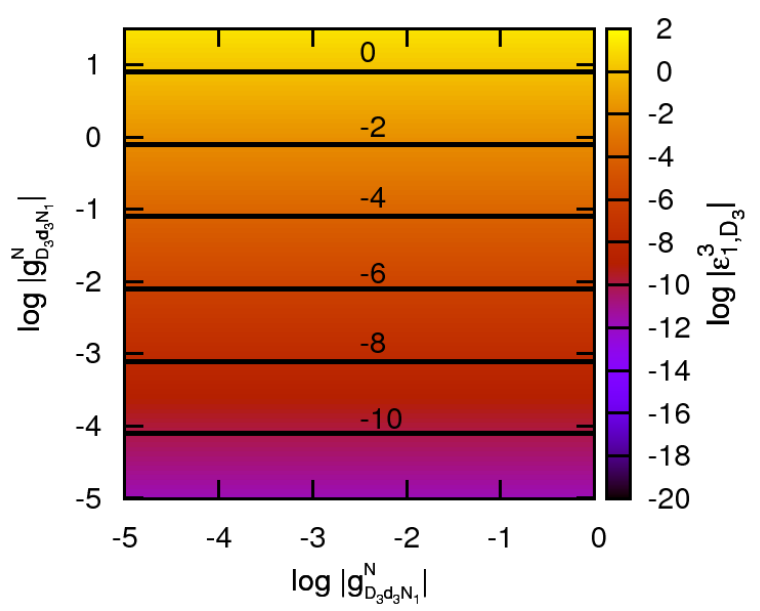

(b)

Figure 9: Logarithm (base 10) of the maximal values of $\left|\varepsilon_{1, \mu}^{3}\right|=\left|\varepsilon_{1, \tau}^{3}\right|(a)$ and $\left|\varepsilon_{1, D_{3}}^{3}\right|$ (b) in the $\mathrm{E}_{6} \mathrm{SSM}$ Model II versus $\log \left|g_{D_{3} d_{3} N_{2}}^{N}\right|$ and $\log \left|g_{D_{3} d_{3} N_{1}}^{N}\right|$ for $M_{2}=10 M_{1}$ (fixing the ratio $M_{1} / M_{2}$ these asymmetries become independent of $\left.M_{1}\right)$. All couplings $\left|h_{H_{k}^{u} L_{4} N_{j}}^{N}\right|$ and $\left|h_{H_{\alpha}^{u} L_{x} N_{j}}^{N}\right|(\alpha=1,2)$ are set to zero. The solid contour lines show steps of 2 in the logarithm (base 10) of the asymmetries. 